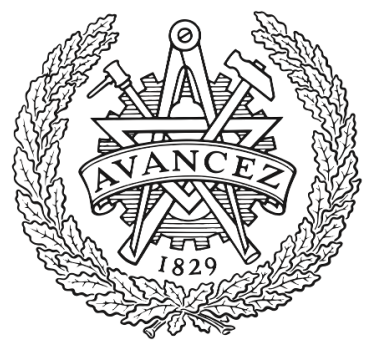

CHALMERS

UNIVERSITY OF TECHNOLOGY

\title{
Thin reaction zones in constant-density turbulent flows at low Damköhler numbers: Theory and simulations
}

Downloaded from: https://research.chalmers.se, 2023-04-26 10:51 UTC

Citation for the original published paper (version of record):

Sabelnikov, V., Yu, R., Lipatnikov, A. (2019). Thin reaction zones in constant-density turbulent flows at low Damköhler numbers: Theory and simulations. Physics of Fluids, 31(5): 1-19. http://dx.doi.org/10.1063/1.5090192

N.B. When citing this work, cite the original published paper. 
 \\ Thin reaction Z/9nefanuscript was accepted by Phys. Fluids. Click here to see the version of record. AIP/123-QED}

Publishiाigin reaction zones in constant-density turbulent flows at low Damköhler numbers: theory and simulations

V.A. Sabelnikov, ${ }^{1}$ R. Yu, ${ }^{2}$ and A.N. Lipatnikov ${ }^{3}$ a)

${ }^{1)}$ ONERA - The French Aerospace Lab., F-91761 Palaiseau, France $^{\text {b) }}$

${ }^{2)}$ Division of Fluid Mechanics, Lund University, Lund, 22100 , Sweden

${ }^{3)}$ Department of Mechanics and Maritime Sciences, Chalmers University of Technology, Göteborg, 412 96, Sweden

(Dated: 15 April 2019)

Propagation of a single-reaction wave in a constant-density turbulent flow is studied by considering reaction rates that depend on the reaction progress variable $c$ in a highly nonlinear manner. Analysis of Direct Numerical Simulation (DNS) data obtained recently from 26 reaction waves characterized by low Damköhler $(0.01<D a<1)$ and high Karlovitz $(6.5<K a<587)$ numbers reveals the following trends. First, the ratio of consumption velocity $U_{T}$ to rms turbulent velocity $u^{\prime}$ scales as square root of $D a$ in line with Damköhler's classical hypothesis. Second, the ratio of fully-developed turbulent wave thickness to an integral length scale of turbulence decreases with increasing $D a$ and tends to scale with inverse square root of $D a$, in line with the same hypothesis. Third, contrary to the widelyaccepted concept of distributed reaction zones, reaction-zone broadening is quite moderate even at $D a=0.01$ and $K a=587$. Fourth, contrary to the same concept, $U_{T} / u^{\prime}$ is mainly controlled by reaction-surface area. Fifth, $U_{T} / u^{\prime}$ does not vary with laminar-reaction-zone thickness provided that $D a$ is constant. To explain the totality of these DNS results, a new theory is developed by (i) exploring the propagation of a molecular mixing layer attached to an infinitely thin reaction sheet in a highly turbulent flow and (ii) hypothesizing that the area of the reaction sheet is controlled by turbulent mixing. This hypothesis is supported by order-of-magnitude estimates and results in the aforementioned Damköhler's scaling for $U_{T} / u^{\prime}$. The theory is also consistent with other aforementioned DNS results and, in particular, explains the weak influence of the laminar-reaction-zone thickness on $U_{T} / u^{\prime}$.

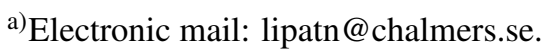

b) Also at Central Aerohydrodynamic Institute (TsAGI), 140180 Zhukovsky, Moscow Region, Russian Federation 
The problem of the influence of turbulence on reaction waves is directly relevant to various phenomena ranging from reactions in aqueous solutions ${ }^{1}$, turbulent combustion ${ }^{2-4}$, or deflagration-todetonation transition ${ }^{5}$ under terrestrial conditions to evolution of thermonuclear Ia supernovae ${ }^{6-8}$ in the Universe. This highly nonlinear and multiscale fundamental problem attracted much attention since the 1940 s when significant flame acceleration by turbulence was discovered. The effect was first explained by Damköhler ${ }^{9}$ who hypothesized two limiting regimes of the influence of turbulence on combustion depending on the ratio of an integral turbulence length scale $L$ to laminar flame thickness $\delta_{L}$. Subsequently, various regimes of the influence of turbulence on a reaction wave were widely discussed and several regime diagrams were proposed by considering self-propagation of a dynamically passive reaction wave in constant-density turbulence ${ }^{10-12}$. For brevity, these regime diagrams will be called Classical Regime Diagrams (CRDs) in the following.

Despite strong simplifications, the regime-diagram approach has clearly demonstrated importance for understanding of fundamental mechanisms governing more complex phenomena. Accordingly, the CRDs are widely accepted as an appropriate framework for speculating what physical mechanisms govern the influence of turbulence on a reaction wave and under what conditions. In particular, "oversimplified" CRDs are discussed in almost every second archival publication on premixed turbulent combustion, even though important effects such as (i) preferential diffusion $^{2,4,13-15}$, (ii) variable density ${ }^{16-19}$, and (iii) complex chemistry ${ }^{20-22}$ are well documented for flames but disregarded in the CRDs (certain recent combustion-regime diagrams ${ }^{4,23-26}$ allow for thermal expansion effects). It is also worth noting that a regime of premixed turbulent burning that was controlled by combustion chemistry was hypothesized by Shetinkov ${ }^{27}$ as early as 60 years ago.

While the CRDs are well accepted and widely used, there still remains a challenging area of research: reaction waves at high turbulent Reynolds numbers

$$
R e_{t}=\frac{u^{\prime} L}{v} \gg 1
$$

low Damköhler numbers

$$
D a=\frac{\tau_{T}}{\tau_{L}}<1,
$$

and correspondingly high Karlovitz numbers

$$
K a=\frac{\tau_{L}}{\tau_{K}}=S c\left(\frac{\delta_{L}}{\eta_{K}}\right)^{2}=S c^{-1}\left(\frac{v_{K}}{S_{L}}\right)^{2}=\frac{R e_{t}^{1 / 2}}{D a} \gg 1
$$


Publishihgre, rms velocity $u^{\prime}$, integral length scale $L$, and time scale $\tau_{T}=L / u^{\prime}$ characterize large turbulent eddies; the Kolmogorov microscales $v_{K}=\eta_{K} / \tau_{K}, \eta_{K}=\left(v^{3} / \bar{\varepsilon}\right)^{1 / 4}$, and $\tau_{K}=(v / \bar{\varepsilon})^{1 / 2}$ characterize the smallest turbulent eddies; $\tau_{L}=\delta_{L} / S_{L}, \delta_{L}=D / S_{L}$, and $S_{L}$ are the laminar-wave time scale, thickness, and speed, respectively; $v$ is the kinematic viscosity of the fresh mixture; $D$ is the molecular diffusivity of the deficient reactant; $S c=v / D$ is the Schmidt number; $\bar{\varepsilon}=2 \overline{v S_{i j} S_{i j}} \propto u^{\prime 3} / L$ is the mean dissipation rate ${ }^{28,29}$; and $S_{i j}=0.5\left(\partial u_{i} / \partial x_{j}+\partial u_{j} / \partial x_{i}\right)$ is the rate-of-strain tensor. Henceforth, the summation convention applies to repeated indexes.

Indeed, on the one hand, by referring to the pioneering work by Damköhler ${ }^{9}$ and Shchelkin ${ }^{30}$, a Distributed Reaction Zone (DRZ) regime was hypothesized at $D a<1$ and $\operatorname{Re}_{t} \gg 1$ in CRDs ${ }^{10-12}$. In that regime, reaction zones are said to be "thickened"10, "distributed" "11, "well-stirred"12, or "broadened"31.

On the other hand, it is difficult to find a direct evidence of DRZs in intense turbulence. In particular, the DRZ concept is often supported by experimental and DNS data that yield

$$
S_{T} \propto S_{L} \operatorname{Re}_{t}^{1 / 2} \propto u^{\prime} D a^{1 / 2}
$$

for turbulent reaction-wave speed. In the case of $L \ll \delta_{L}$ and $S c=\mathrm{O}(1)=$ const, this scaling was first predicted by Damköhler ${ }^{9}$ by reducing the influence of turbulence on a reaction wave to intensification of mixing within reaction waves. Subsequently, Eq. (4) was confirmed (i) in experiments on reaction-front propagation in aqueous solutions ${ }^{1}$ and flames at low $D a^{32}$ and (ii) in 2D DNSs of constant-density reaction waves ${ }^{33}$, 3D DNSs of thermonuclear deflagration ${ }^{8}$, and 3D DNSs of lean methane-air and hydrogen-air flames ${ }^{34}$. However, strictly speaking, validation of Eq. (4) is not sufficient evidence in favor of the DRZ concept, because the same scaling was also obtained by developing alternative approaches, e.g., see a recent paper by Chaudhuri et al. ${ }^{26}$ At the same time, statistically significant reaction-zone broadening is rarely documented for reactions whose rates vary in a highly nonlinear manner across the wave (e.g., with temperature in a flame), as discussed in detail in Section IIC 1.

Thus, characterization of a single-reaction zone in a constant-density turbulent flow at $D a<1$ and $K a \gg 1$ is still of fundamental interest. This paper reports the results of a study originally conceived with a view to numerically exploring the eventual reaction-zone broadening and examining the scaling given by Eq. (4) under a wide range of conditions such that $D a<1$ and $K a \gg 1$. However, the DNS data obtained were not fully expected: Eq. (4) was very well validated, but no statistically significant reaction-zone broadening was detected. As a consequence, we expanded 
Publishiting scope of our work to include the development of a model consistent with the DNS data.

The three aforementioned tasks appear to be of fundamental interest in themselves. Moreover, they are directly relevant to the various phenomena mentioned in the beginning of this section, e.g. to premixed turbulent flames. Since the problem at hand is relatively simple, a DNS study can be performed for a much wider range of conditions than those amenable to a 3D DNS of turbulent burning with complex chemistry. Furthermore, interpretation of results, and particularly model development, is much easier when considering the propagation of a single-reaction wave in constant-density turbulence. At the same time, since the mechanisms underlying turbulence effects on the wave are also of importance for premixed turbulent combustion, results presented below could also contribute to better understanding highly turbulent premixed flames. Indeed, the influence of turbulence on a reaction-wave surface and structure is an important ingredient of flame-turbulence interaction and a submodel of the former phenomenon is a basic building block for a typical model of premixed turbulent combustion ${ }^{2-4,10,11,24-26}$. Even if effects due to preferential diffusion ${ }^{2,4,13-15}$, variable density ${ }^{16-19}$, and complex chemistry ${ }^{20-22}$ are well documented for turbulent burning but ignored in the present study, target-directed investigation of the influence of turbulence on a reaction-wave under the simplest conditions has its own value. Nevertheless, bearing in mind the above reseryations, care should be taken when applying the results reported in the following to premixed turbulent combustion.

The last remark motivates adding a supplementary goal to this work. Since there are both common features and distinctions between the propagation of a single-reaction wave in constantdensity turbulence and premixed turbulent burning, it is of interest to explore what phenomena observed in flames can (or cannot) be qualitatively predicted by investigating the greatly simplified model outlined above. Accordingly, in the rest of the paper, results of the present study will often be compared with published results obtained from premixed turbulent flames in order to clarify common and distinctive points. The focus of the comparison will be placed on highly turbulent flames characterized by $K a \gg 1$ and $D a<1$. Such conditions were realized in recent laboratory experiments with (i) piloted Bunsen flames studied in Michigan ${ }^{31,35-38}$, (ii) piloted jet flames studied in Lund ${ }^{39-42}$, Sydney ${ }^{43}$, or Sandia ${ }^{44}$, as well as counter-flow reactant-product flames. In fact, over the past decade, the focus of experimental research into premixed turbulent combustion was strongly shifted to these flame configurations, because they offer an opportunity to study burning under conditions of high (low) Karlovitz (Damköhler) numbers. Such flames are of great practical importance because burning in combustion chambers of modern gas turbines is often 
The paper is organized as follows. In the next section, recent DNS data ${ }^{45-51}$ are analyzed in order (i) to explore eventual broadening of reaction zones by small-scale turbulent eddies in the case of $D a<1$ and $K a \gg 1$, (ii) to test Eq. (4), and (iii) to gain further insight into physical mechanisms responsible for the increase in consumption velocity due to turbulence. In the third section, to explain the DNS data, a simple phenomenological theory of the influence of constantdensity turbulence on propagation of an infinitely thin reaction zone is developed for the case of $D a<1$ and $K a \gg 1$ and Eq. (4) is derived. Conclusions are summarized in the fourth section.

\section{DIRECT NUMERICAL SIMULATIONS}

\section{A. DNS attributes}

Because the simulations analyzed in the following were discussed in detail in recent papers ${ }^{45-51}$, we restrict ourselves to a summary of the governing equations, numerical methods, and turbulence and mixture characteristics. The reader interested in details is referred to the cited papers.

\section{Equations}

The background turbulent flow is described by the continuity and Navier-Stokes equations

$$
\begin{gathered}
\nabla \cdot \boldsymbol{u}=0, \\
\frac{\partial u}{\partial t}+(\boldsymbol{u} \cdot \nabla) \boldsymbol{u}=-\rho^{-1} \nabla p+v \nabla^{2} \boldsymbol{u}+\boldsymbol{f},
\end{gathered}
$$

where $t$ is time, $\boldsymbol{x}=\{x, y, z\}$ is the spatial coordinate vector, $\boldsymbol{u}=\{u, v, w\}$ is the flow velocity vector, the density $\rho$ and viscosity $v$ are constant, $p$ is pressure, and a vector-function $f$ is added in order to maintain a constant turbulence intensity by using forcing at low wavenumbers.

Propagation of a reaction wave is modeled by the convection-diffusion-reaction equation

$$
\frac{\partial c}{\partial t}+\boldsymbol{u} \cdot \nabla c=D \nabla^{2} c+W
$$

for reaction progress variable $c$, i.e., the mass fraction of a reactant or product species normalized so that $c=0$ and 1 in unburned reactants and equilibrium products, respectively. Here, the molecular diffusivity $D$ is constant and the reaction rate

$$
W=\frac{1}{1+\tau} \frac{1-c}{\tau_{R}} \exp \left[-\frac{Z e(1+\tau)^{2}}{\tau(1+\tau c)}\right]
$$


Publishilisg highly nonlinear function of $c$, because we use $Z e=6.0$ or 17.1 and $\tau=6.0$. As discussed in detail elsewhere ${ }^{49}$, Eq. (8) allows us to mimic the behavior of the reaction rate in a flame by considering constant-density reacting flows. Before each DNS run, the values of $S_{L}$ and $\delta_{L}$ were set and the required values of $D$ and a reaction time scale $\tau_{R}$ were determined in pre-simulations of the planar 1D laminar reaction wave modeled by Eqs. (7) and (8).

\section{Numerical setup and methods}

The computational domain was a rectangular box of size of $\Lambda_{x} \times \Lambda \times \Lambda$ and was discretized using a uniform staggered Cartesian grid of $N_{x} \times N \times N$ cells. Most results reported in the following were obtained using three different domains (three different $\Lambda$ ), but retaining the same spatial resolution $\Delta x=\Delta y=\Delta z=\Lambda_{x} / N_{x}=\Lambda / N$.

The boundary conditions were periodic not only in the transverse directions $y$ and $z$, but also in the direction $x$ normal to the mean wave surface. Accordingly, when the reaction wave reached the left boundary $(x=0)$ of the computational domain, an identical reaction wave entered the domain at the right boundary $\left(x=\Lambda_{x}\right)$. Such a method ${ }^{46}$ allowed us to greatly improve sampling statistics by simulating a number of cycles of wave propagation through the computational domain.

At the end $t=t_{1}$ of the time interval $\left(0, t_{1}\right)$ required to generate fully-developed, homogeneous, isotropic turbulent field, see Section II A 3, a plane wave $c(\boldsymbol{x}, t=0)=c_{L}(\xi)$ was released at $x^{0}=\Lambda_{x} / 2$ such that $\int_{-\infty}^{0} c_{L}(\xi) \mathrm{d} \xi=\int_{0}^{\infty}\left[1-c_{L}(\xi)\right] \mathrm{d} \xi$ and $\xi=x-x^{0}$. Here, $c_{L}(\xi)$ is the precomputed laminar-wave profile with $\mathrm{d} c / \mathrm{d} \xi \geq 0$.

Simulations were performed using a simplified in-house DNS solver ${ }^{52}$ developed for lowMach-number reacting flows and equipped with a standalone stiff chemistry solver for a general kinetic mechanism. For the goals of the present work, the solver was simplified and temporal advancement by a full time-step $\left(\Delta t=t^{n+1}-t^{n}=0.029 \Delta x / u^{\prime}\right)$ was performed using the AdamsBashforth method in multiple sub-time steps, as described by Yu and Lipatnikov ${ }^{47}$. The convection term in Eq. (7) was discretized using a fifth order Weighted Essentially Non-Oscillatory (WENO) scheme $^{53}$, whereas all other spatial terms were discretized using sixth order centered schemes. The pressure field was computed by integrating the constant-coefficient Poisson equation with the help of an accurate spectrum solver using an open source, parallel version of FFTW3 (mpi-fftw). The DNS code is implemented in vector form enabling 1D, 2D, and 3D simulations. 
TABLE I. Six turbulence fields

\begin{tabular}{cccccccccccc}
\hline Field & $R e_{0}$ & $N_{x} \times N \times N$ & $\frac{L_{k \varepsilon}}{\Lambda}$ & $R e_{k \varepsilon}$ & $\frac{L_{11}}{\Lambda}$ & $R e_{t}$ & $\frac{\eta_{K}}{\Delta x}$ & $\frac{\tau_{T}}{\tau_{T}^{0}}$ & $\frac{\tau_{T}}{\tau_{K}}$ & $\frac{L_{11}}{\eta_{K}}$ & $\frac{L_{k \varepsilon}}{\eta_{K}}$ \\
A & 50 & $256 \times 64^{2}$ & 0.20 & 41 & 0.13 & 26 & 0.68 & 0.51 & 5.5 & 12 & 18 \\
B & 100 & $512 \times 128^{2}$ & 0.26 & 105 & 0.12 & 49 & 0.86 & 0.47 & 6.2 & 17 & 37 \\
C & 200 & $1024 \times 256^{2}$ & 0.30 & 241 & 0.11 & 84 & 1.06 & 0.42 & 7.4 & 25 & 68 \\
D & $،$ &, & 0.71 & 566 & 0.20 & 157 & 1.32 & 0.79 & 9.1 & 38 & 135 \\
E & $،$ &, & 0.14 & 114 & 0.06 & 50 & 0.88 & 0.25 & 6.4 & 18 & 42 \\
F & 50 &, & 0.20 & 41 & 0.13 & 26 & 2.69 & 0.51 & 5.5 & 12 & 18 \\
\hline \hline
\end{tabular}

\section{Turbulence generation}

The initial turbulence field was generated by synthesizing prescribed Fourier waves ${ }^{54}$ with an initial rms velocity $u_{0}^{\prime}$ and a turbulence length scale $L_{0}=\Lambda / 4$. The forcing function $f$ introduced into Eq. (6) to maintain statistically stationary turbulence was specified adapting the methods proposed by Eswaran and Pope 55 and Lamorgese et al. ${ }^{56}$, as discussed in detail by $\mathrm{Yu}$ and Lipatnikov ${ }^{47}$. After a transient phase, at $t>t_{1}=5 \tau_{T}^{0}=5 L_{0} / u_{0}^{\prime}$, the generated turbulence was homogeneous, see Fig. 2 in Ref. 45, isotropic, see Fig. 1 in Ref. 47, and statistically stationary, see Fig. 2 in Ref. 48, with volume-averaged values of $u^{\prime}$ or the dissipation rate $\varepsilon$ being very close to $u_{0}^{\prime}$ or weakly oscillating around $1.6 u_{0}^{\prime 3} / L_{0}$, respectively, see Fig. 1 in Ref. 46.

Most results reported in the following were obtained by adapting one of the basic turbulence fields $\mathrm{A}, \mathrm{B}$, and $\mathrm{C}$ generated by setting three different values for the width $\Lambda$ of the computational domain and hence three different "initial" turbulent Reynolds numbers $R e_{0}=u_{0}^{\prime} L_{0} / v=50$, 100 , or 200 . The characteristics of the three turbulence fields, calculated by averaging the dissipation rate $\varepsilon(x, t)$ and various moments of the velocity field over the computational volume and time at $t>t_{1}$, are listed in Table I. Here, the rms velocity $u^{\prime}=(\overline{\langle\boldsymbol{u} \cdot \boldsymbol{u}\rangle} / 3)^{1 / 2}, L_{11}=$ $\int_{0}^{L / 2} R_{11}^{u}(r) d r \approx \int_{0}^{L / 2} R_{11}^{v}(r) d r \approx \int_{0}^{L / 2} R_{11}^{w}(r) d r$ and $\tau_{T}=L_{11} / u^{\prime}$ are the longitudinal integral length scale and the corresponding time scale, respectively; $R_{11}^{u}(r)=\overline{\langle u(x, y, z) u(x+r, y, z)\rangle} / u^{\prime 2}, R_{11}^{v}(r)=$ $\overline{\langle v(x, y, z) v(x, y+r, z)\rangle} / u^{\prime 2}$, and $R_{11}^{w}(r)=\overline{\langle w(x, y, z) v(x, y, z+r)\rangle} / u^{\prime 2}$ are auto-correlation functions vanishing at $r=\Lambda / 2$ in all three cases, see Fig. 1 in Ref. $47 ; L_{k \varepsilon}=(\overline{\langle\boldsymbol{u} \cdot \boldsymbol{u}\rangle} / 2)^{3 / 2} \overline{\langle\boldsymbol{\langle \varepsilon}\rangle}$ is another turbulence length scale often used in DNS papers; $R e_{t}=u^{\prime} L_{11} / v$ and $R e_{k \varepsilon}=u^{\prime} L_{k \varepsilon} / v$ are the Reynolds numbers based on the two length scales; and $\overline{\langle q\rangle}$ designates the value of the quantity $q$ 
Publishiange aged over the computational volume and time at $t>t_{1}$.

Adapting the method proposed by Lamorgese et al. ${ }^{56}$, we generated ${ }^{47}$ two more turbulence fields, D and E, by changing spectral characteristics of the forcing function $f$ in order to vary the ratio $L_{11} / \Lambda$ while retaining the same $u^{\prime}$ and $v$. The generated turbulence fields are ranked in descending order of the spectral width of the $-5 / 3$ slope range ${ }^{28,29}$ as follows, see Fig. 3 in Ref. 48 : $\mathrm{D}>\mathrm{C}>\mathrm{B}$ and $\mathrm{E}>\mathrm{A}$, in line with the values of $R e_{k \varepsilon}$ given in Table I.

For each velocity field, the Kolmogorov length scale $\eta_{K}$ was on the order of the grid cell size $\Delta x$, thus implying sufficient grid resolution. Nevertheless, to confirm the low sensitivity of results to grid resolution, field $\mathrm{F}$ was generated, with the same $f$ and $\Lambda$ as in case A but with $\Delta x / \eta_{K}$ smaller by a factor of four.

\section{Studied cases}

Various cases were set up by selecting a turbulence field and specifying $S_{L}, \delta_{L}$, and $Z e$. Since the reaction wave did not affect the flow, the choice of a turbulence field was independent of the choice of $S_{L}, \delta_{L}$, and $Z e$. The values of $D, \tau_{R}$, and $S c$ required to obtain the specified $S_{L}$ and $\delta_{L}$ were found in $1 \mathrm{D}$ pre-computations of the laminar wave. Overall 45 cases characterized by $D a=0.01-24.7, K a=0.36-587, u^{\prime} / S_{L}=0.5-90$, and $L / \delta_{L}=0.39-12.4$ were simulated, including cases being designed to demonstrate the weak sensitivity of computed results to grid resolution, $L_{11} / \Lambda$, etc. Reasons for selecting each of the 45 cases were discussed elsewhere ${ }^{48}$.

In the following, we consider only the 26 cases where $D a<1$. Note that case K4, with $D a=$ 0.01 , was omitted in the present study, because the viscosity in that case was higher compared to other 44 cases and, consequently, $R e_{t}=14$ was too low. Nevertheless, the results computed in case K4 are consistent with those obtained in other 26 cases with $D a<1$. The characteristics of these 26 cases are summarized in Table II. In certain cases, the length $\Lambda_{x}$ of the computational domain was increased in order for the total thickness of the reaction wave brush to be significantly less than $\Lambda_{x}$. To retain the same resolution, the number $N_{x}$ of grid points in the axial direction was increased accordingly. 
TABLE II. Studied cases

\begin{tabular}{|c|c|c|c|c|c|c|c|c|c|c|}
\hline & Field & $\frac{\Lambda_{x}}{\Lambda}$ & $N_{x}$ & $Z e$ & $S c$ & $D a$ & $K a$ & $\frac{u^{\prime}}{S_{L}}$ & $\frac{L_{11}}{\delta_{L}}$ & $\frac{L_{k \varepsilon}}{\delta_{L}}$ \\
\hline B4 & A & 4 & 256 & 6.0 & 0.39 & 0.41 & 13.4 & 5 & 2.1 & 3.2 \\
\hline B5 & ' & ' & " & ' & 0.78 & 0.20 & 26.9 & 10 & $،$ & " \\
\hline B9 & B & $،$ & 512 & " & 0.39 & 0.75 & & 5 & 3.7 & 8.0 \\
\hline B10 & ، & $،$ & ' & ' & 0.78 & 0.38 & & 10 & $،$ & ' \\
\hline B15 & $\mathrm{C}$ & $،$ & 1024 & ، & 0.78 & 0.67 & & 10 & 6.7 & 18.2 \\
\hline D1 & ' & 8 & 2048 & $،$, & ' & 0.17 & & 20 & 3.46 & 9.4 \\
\hline D2 & ، & ' & ' & ' & ' & 0.08 & & 30 & 2.35 & 6.4 \\
\hline D3 & ' & ' & " & ، & & 0.0 & 173. & 40 & 1.76 & 4.8 \\
\hline D4 & ' & $،$ & $،$ & ' & & 0.02 & 390. & 60 & 1.16 & 3.2 \\
\hline D5 & B & ' & 1024 & & & 0.97 & 6.53 & 2 & 1.95 & 4.2 \\
\hline D6 & ' & ' & & & & 0.16 & 40.8 & 5 & 0.78 & 1.7 \\
\hline D7 & ' & ', & & & & 0.04 & 163. & 10 & 0.39 & 0.85 \\
\hline D8 & ، & $،$ & & & & 0.04 & 147. & 30 & 1.29 & 2.8 \\
\hline D9 & " & ،, & & & $،$ & 0.01 & 587. & 60 & 0.65 & 1.4 \\
\hline D10 & A & ،, & & & '" & 0.02 & 238. & 30 & 0.70 & 1.1 \\
\hline $\mathrm{T} 2$ & $\mathrm{C}$ & 4 & & & 0.24 & 0.21 & 36.2 & 10 & 2.1 & 5.7 \\
\hline $\mathrm{T} 3$ & , & & & $،$ & 3.13 & 0.08 & 97.4 & 60 & 4.67 & 12.7 \\
\hline $\mathrm{T} 4$ & & & & ،, & 7.04 & 0.08 & 97.6 & 90 & 6.94 & 18.9 \\
\hline T5 & & & 1024 & ' & 0.03 & 0.67 & 11.0 & 2 & 1.34 & 3.7 \\
\hline T6 & & & $،$ & ' & 0.20 & 0.68 & 11.0 & 5 & 3.39 & 9.3 \\
\hline K3 & & & 2048 & ' & 28.2 & 0.09 & 62.1 & 90 & 8.27 & 12.7 \\
\hline H1 & & 4 & 1024 & ' & 0.78 & 0.21 & 27.7 & 10 & 2.1 & 3.2 \\
\hline & & ', & ', & 17.1 & $،$ & $،$ & ' & $،$, & $،$ & ' \\
\hline F & $\mathrm{C}$ & ،, & ' & ' & 0.24 & $،$ & 36.2 & $،$ & ' & 5.7 \\
\hline H4 & $،$ & 8 & 2048 & ' & 0.78 & 0.02 & 389. & 60 & 1.16 & 3.2 \\
\hline H5 & $\mathrm{E}$ & 4 & 1024 & 6.0 & ' & 0.39 & 16.1 & 10 & 3.88 & 9.1 \\
\hline
\end{tabular}




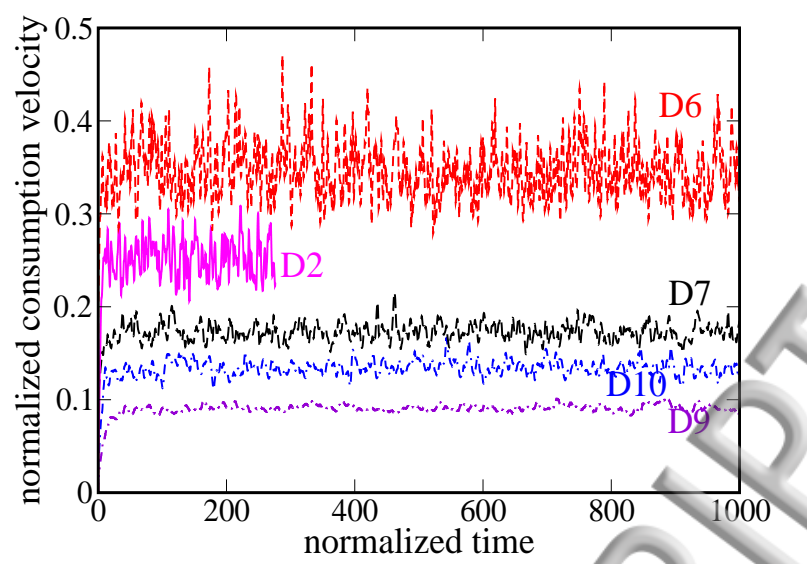

FIG. 1. Normalized instantaneous turbulent consumption velocity $\hat{U}_{T}(t) / u^{\prime}$ vs. normalized time $t / \tau_{T}$. Cases are indicated at curves.

\section{Sampled statistics}

Statistics were sampled using the following methods. First, for each characteristic $q(\boldsymbol{x}, t)$ of the reaction wave $c(\boldsymbol{x}, t)$, its time-dependent "mean" value $\hat{q}(x, t)$ was evaluated by averaging the DNS data over transverse coordinates $y$ and $z$, and the instantaneous turbulent consumption velocity $\hat{U}_{T}(t)$ was calculated as follows

$$
\hat{U}_{T}(t)=\int_{0}^{\Lambda_{x}} \hat{W}(x, t) d x .
$$

Second, computations of fully developed statistics were started after the end $t_{2}$ of a transient phase characterized by substantial and almost monotonic increase in $\hat{U}_{T}(t)$. The duration $t_{2}-t_{1}$ of the transient phase varied from case to case and showed a trend to an increase with decreasing $D a$. However, analysis of data on this duration is beyond the scope of the present work. At $t>t_{2}$, sampling was performed at every 100th full time step over a time interval of $t_{3}-t_{2}>50 \tau_{T}^{0}$, with $t_{3}-t_{2}$ being as long as $1500 \tau_{T}$ in some cases. During that time interval, the normalized turbulent consumption velocity $\hat{U}_{T}(t) / u^{\prime}$ oscillated around its fully-developed value $U_{T} / u^{\prime}$ (see Fig. 1). Fully-developed mean quantities $\bar{q}(x)$ and $U_{T}$ were evaluated by averaging $\hat{q}(x, t)$ and $\hat{U}_{T}(t)$, respectively, over time within $\left(t_{2}, t_{3}\right)$.

Moreover, at $t_{2} \leq t \leq t_{3}$, 3D joint Probability Density Functions (PDFs) $P(c, q, \bar{c})$ were computed for various quantities $q$ such as $|\nabla c|$ in the run-time mode, i.e., each grid cell and each sampling time step contributed to the PDFs, as discussed in detail by Yu and Lipatnikov ${ }^{47}$. Using 

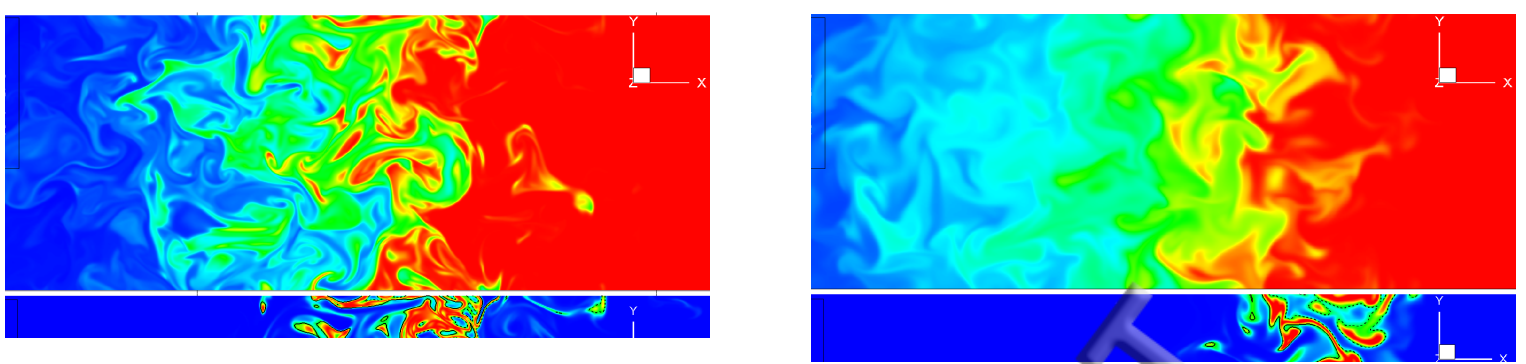

(a)

(b)

FIG. 2. Typical 2D images of the reaction progress variable $c(\boldsymbol{x}, t)$ (top row) and normalized reaction rate $W[c(\boldsymbol{x}, t)]$ (bottom row) in an $x-y$ slice plane. The rate is normalized to the maximum value $\max \{W(c)\}$ of the function $W(c)$ defined by Eq. (8), where $\tau, \tau_{R}$ and Ze are kept constant. Black dashed and solid lines represent boundaries of the reaction zone, i.e. $c(\boldsymbol{x}, t)=c_{r, 1}$ and $c_{r, 2}$, respectively. The thickness $\delta_{r}$ is equal to the distance between the planes $c(\boldsymbol{x}, t)=c_{r, 1}$ and $c(\boldsymbol{x}, t)=c_{r, 2}$ in the unperturbed laminar reaction wave. (a) Case T4, Ze =6.0, Da=0.08, and $K a=97.6$, (b) Case H4, Ze=17.1, Da=0.02, and $K a=389$.

such joint PDFs, values of $q$ conditioned to an interval $\left(c_{1}, c_{2}\right)$ were evaluated as follows:

$$
\langle q\rangle_{c 1, c 2}=\int_{-\infty}^{+\infty} \int_{0}^{1} \int_{c_{1}}^{c_{2}} q P(q, c, \bar{c}) \mathrm{d} c \mathrm{~d} \bar{c} \mathrm{~d} q / \int_{-\infty}^{+\infty} \int_{0}^{1} \int_{c_{1}}^{c_{2}} P(q, c, \bar{c}) \mathrm{d} c \mathrm{~d} \bar{c} \mathrm{~d} q .
$$

In particular, two sets of reaction-zone boundaries were used; (i) $c_{1}=c_{r, 1}$ and $c_{2}=c_{r, 2}$ such that $W\left(c_{r, 1}\right)=W\left(c_{r, 2}\right)=\max \{W(c)\} / 2$ and $c_{r, 1}<c_{r, 2}$ or (ii) $c_{1}=c_{w}-0.005$ and $c_{2}=c_{w}+0.005$, where $c_{w}$ is defined by $W\left(c_{w}\right)=\max \{W(c)\}$. In the following, a quantity $q$ averaged over a thicker reaction zone (i) and a thinner reaction zone (ii) are denoted by $\langle q\rangle_{r}$ and $\langle q\rangle_{w}$, respectively.

\section{B. Results and discussion}

Typical 2D slices of 3D fields of reaction progress variable $c(\boldsymbol{x}, t)$ and reaction rate $W(\boldsymbol{x}, t)$ are shown in Fig. 2, and other slices were reported in Fig. 3 in Ref. 47. Both fields are strongly perturbed by turbulence, and both broadened and narrowed reaction zones are observed. Reactionzone broadening seems to be more pronounced in case T4, characterized by a lower $Z e$ but a higher $\mathrm{Da}$ and a lower $\mathrm{Ka}$. In this case, the ratio of the mean reaction-zone thickness to the thickness of 


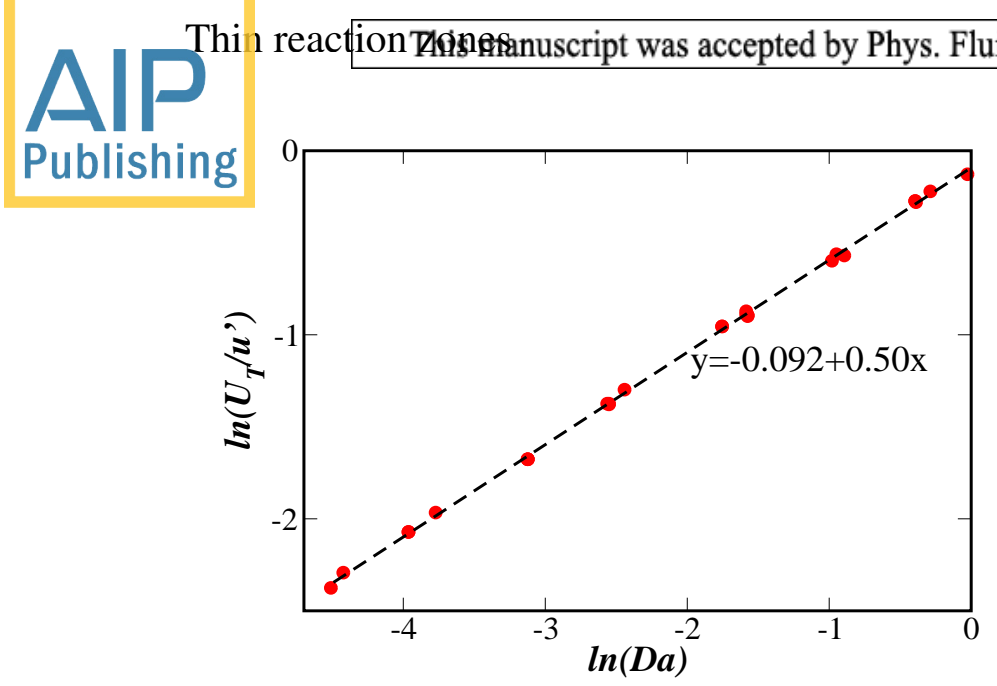

(a)

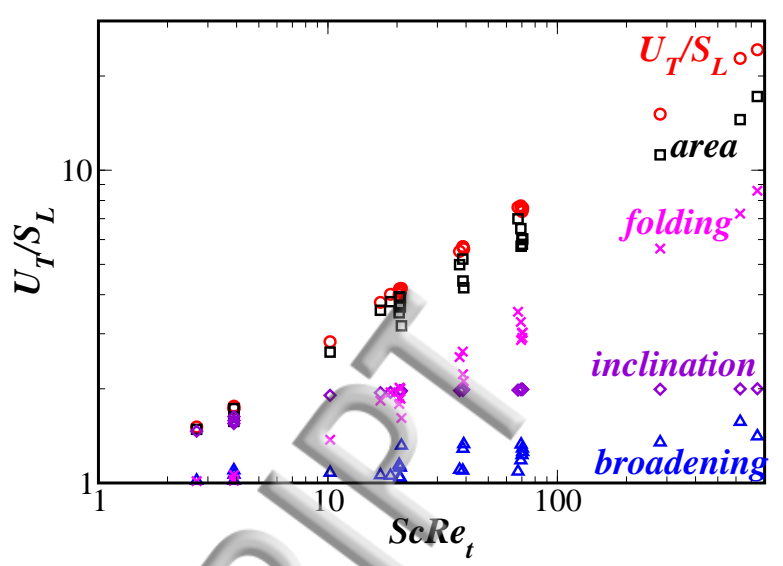

(b)

FIG. 3. (a) Normalized consumption velocity $U_{T} / u^{\prime}$ vs. Damköhler number $D a$. Symbols show DNS data fitted with a straight line. (b) Normalized consumption velocity $U_{T} / S_{L}$ (circles), area increase $\langle\delta A\rangle_{r}$ (squares), and contributions to $\langle\delta A\rangle_{r}$ due to broadening (triangles), inclination (diamonds), and folding (crosses) vs. the product of Schmidt and turbulent Reynolds numbers.

the laminar reaction zone is largest, as will be discussed in Section II B 2, and Fig. 2(a) apparently indicates the broadening effect. It is worth stressing, however, that these images are presented as illustrations. They should not be used to draw conclusions regarding reaction-zone broadening, because inclination of $3 \mathrm{D}$ reaction zones with respect to the $2 \mathrm{D}$ slice plane may significantly increase the apparent thicknesses of the zones. Accordingly, in the rest of the section, we discuss the results of a statistical analysis of the 3D DNS data.

\section{Turbulent consumption velocity}

Figure 3(a) shows that, in all 26 cases characterized by $D a<1$, the computed turbulent consumption velocities (symbols) presented in a log-log plot are very well fitted by a straight line corresponding to Eq. (4), with a correlation coefficient of 0.9997. There are fewer than 26 distinct symbols in Fig. 3(a), because (i) some sets of cases were designed ${ }^{48}$ to keep $u^{\prime}$ and $D a$ constant while varying $L_{11}, S_{L}$, and $\delta_{L}$ and (ii) similar values of $U_{T} / u^{\prime}$ were computed within each of these sets, in line with Eq. (4). If the entire DNS database (27 waves characterized by $D a<1$ and 18 waves characterized by $D a>1$ ) is fitted as $U_{T} \propto u^{\prime} D a^{p}$, then $p=0.48$ but the scatter in computed data is much larger ${ }^{48}$ for $D a>1$.

As noted in Section I, Eq. (4) was earlier validated by experimental data obtained for reaction- 
Publishiffignt propagation in aqueous solutions ${ }^{1}$ and by 2D DNS data $(D a>0.025)$ on constant-density reaction waves ${ }^{33}$. In Section II C 1, Eq. (4) will be further supported by discussing experimental and DNS data obtained from premixed turbulent flames at low Damköhler numbers.

To explore the physical mechanisms responsible for the increase in the normalized consumption velocity $U_{T} / u^{\prime}$ with $D a$, a relative increase in the mean area of the reaction-zone surface was evaluated as

$$
\langle\delta A\rangle_{r}=\frac{1}{\left(t_{3}-t_{2}\right) \Lambda^{2}} \int_{t_{2}}^{t_{3}} \int_{0}^{\Lambda_{x}} \int_{0}^{\Lambda} \int_{0}^{\Lambda} \frac{|\nabla c| \Pi(c)}{c_{r, 2}-c_{r, 1}} \mathrm{~d} \boldsymbol{x} \mathrm{d} t_{2}
$$

where $\Pi(c)=\mathrm{H}\left(c-c_{r, 1}\right)-\mathrm{H}\left(c-c_{r, 2}\right)$ is difference between Heaviside functions, $c_{r, 1}$ and $c_{r, 2}$ are the reaction-zone boundaries. Subsequently, the normalized mean local consumption velocity was estimated as

$$
\frac{\bar{u}_{c}}{S_{L}}=\frac{U_{T}}{\langle\delta A\rangle_{r} S_{L}}
$$

using the DNS data on $U_{T}$ and $\langle\delta A\rangle_{r}$. The ratio $\bar{u}_{c} / S_{L}$ may be larger than unity due to mixing enhancement within reaction zones by small-scale turbulent eddies ${ }^{9}$. However, the ratio may be smaller than unity due to stretching of the zones $^{57}$ by larger but still small-scale eddies ${ }^{13,58,59}$. When $\bar{u}_{c} / S_{L}>1$, the former effect dominates and the zones are statistically broadened, because local consumption velocity is approximately equal to the characteristic reaction rate multiplied by the local reaction-zone thickness. For these reasons, and because $\bar{u}_{c} / S_{L}>1$ in all simulated waves with $D a<1$, the ratio $\bar{u}_{c} / S_{L}$ is referred to here as the magnitude of broadening contribution to $\langle\delta A\rangle_{r}$.

In Fig. 3(b), the values of $\langle\delta A\rangle_{r}$ (squares) are significantly larger than those of $\bar{u}_{c} / S_{L}$ (triangles). Therefore, in all cases studied, the increase in $U_{T} / S_{L}$ is mainly due to an increase in the normalized area $\langle\delta A\rangle_{r}$ of the reaction-zone surface, whereas the increase in $\bar{u}_{c}$ is of substantially less importance even at $D a$ as low as 0.01 . Nevertheless, the DNS data reveal some increase in $\bar{u}_{c}$, which should not be disregarded.

The simulated increase in $\langle\delta A\rangle_{r}$ is explained by two effects; (i) inclination, i.e. deviation of the local normal vector $\boldsymbol{n}$ from the $x$-axis, and (ii) folding, i.e. multiple intersections of reaction zones with a ray normal to the mean wave brush. The magnitudes $\Xi_{n}$ and $\Xi_{f}$ of the contributions of the former and the latter to an increase in $\langle\delta A\rangle_{r}$ can be respectively estimated as

$$
\Xi_{n}=\frac{1}{\left\langle\left|n_{x}\right|\right\rangle_{r}}, \quad \Xi_{f}=\frac{\langle\delta A\rangle_{r}}{\Xi_{n}}
$$




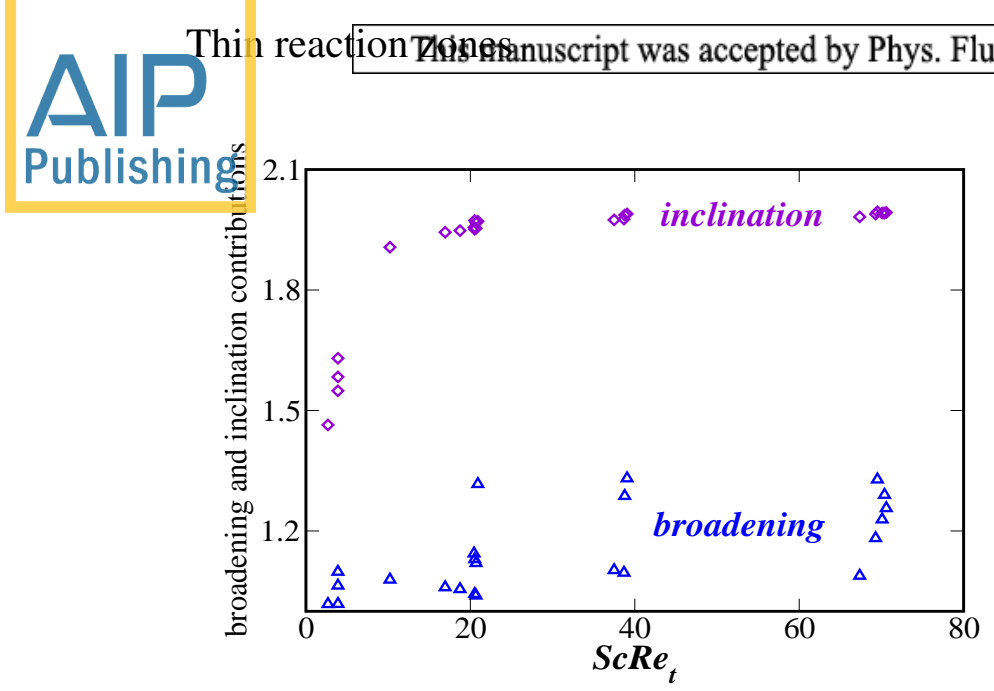

(a)

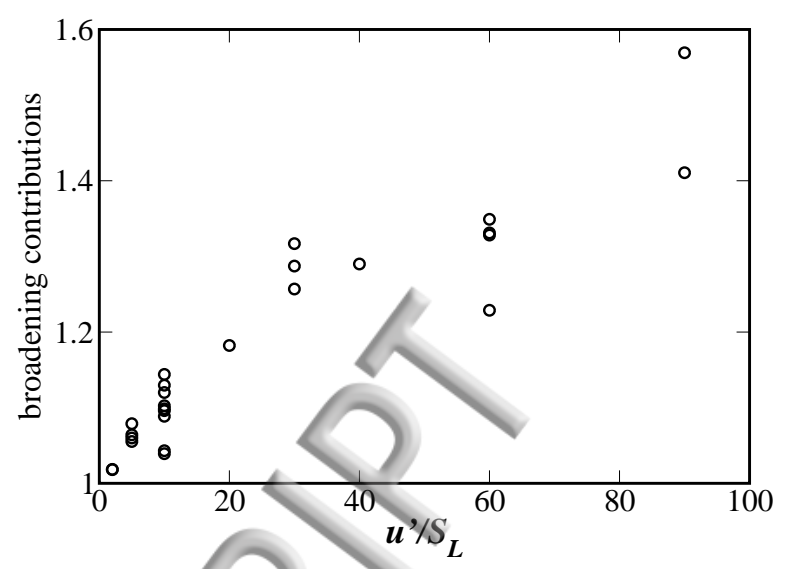

FIG. 4. (a) Broadening (triangles) and inclination (diamonds) contributions vs. product of Schmidt and turbulent Reynolds numbers. (b) Broadening contribution $\bar{u}_{c} / S_{L}$ vs. $u^{\prime} / S_{L}$.

The estimate of the magnitude $\Xi_{f}$ of the folding contribution is based on assumptions that (i) $\langle\delta A\rangle_{r}$ is solely controlled by the inclination and folding effects and (ii) they do not correlate. To verify this estimate, $\Xi_{f}$ was also evaluated as follows

$$
\Xi_{f} \approx \frac{1}{l_{x}\left(t_{3}-t_{2}\right) \Lambda^{2}} \int_{t_{2}}^{t_{3}} \int_{0}^{\Lambda_{x}} \int_{0}^{\Lambda} \int_{0}^{\Lambda} \Pi(c) \mathrm{d} x \mathrm{~d} t
$$

where $l_{x}=\left(c_{r, 2}-c_{r, 1}\right) /\left\langle\left|\nabla_{x} c\right|\right\rangle_{r}$ estimates the mean axial distance between boundaries $c(\boldsymbol{x}, t)=c_{r, 1}$ and $c(x, t)=c_{r, 2}$ of the reaction zone and the integral estimates the mean total $x$-length of all reaction zones. Since Eqs. (13) and (14) yielded similar values of $\Xi_{f}$, solely the former magnitude will be reported in the following.

The diamonds and crosses in Fig. 3(b) indicate that the corresponding $\Xi_{n}$ and $\Xi_{f}$ are comparable at low values of $S c R e_{t}$, but the folding contribution is more significant at larger $S c R e_{t}$. However, $\Xi_{f}$ is only slightly larger than unity in cases D5-D7 and T5. These cases are characterized by a low ratio $S c R e_{t}$ between turbulent and laminar diffusivities (less than four) and a low $u^{\prime} / S_{L}$ or/and $L_{11} / \delta_{L}$. Accordingly, large-scale turbulent eddies are either weak or/and too small and cannot fold reaction zones, while the influence of turbulence on consumption velocity is also weak, $U_{T} / S_{L}<1.8$. Nevertheless, even in these four cases, $U_{T} / S_{L}$ is mainly controlled by $\langle\delta A\rangle_{r}$, whereas the broadening contribution is less than $10 \%, \bar{u}_{c} / S_{L}<1.1$.

The graphs of the broadening and inclination contributions to $U_{T} / S_{L}$ are enlarged in Fig. 4(a). The latter rapidly increases with $S c R e_{t}$ at low values of this product before reaching a plateau around $\Xi_{n}=2$. The broadening contribution is significantly less than $\Xi_{n}$ and weakly increases 


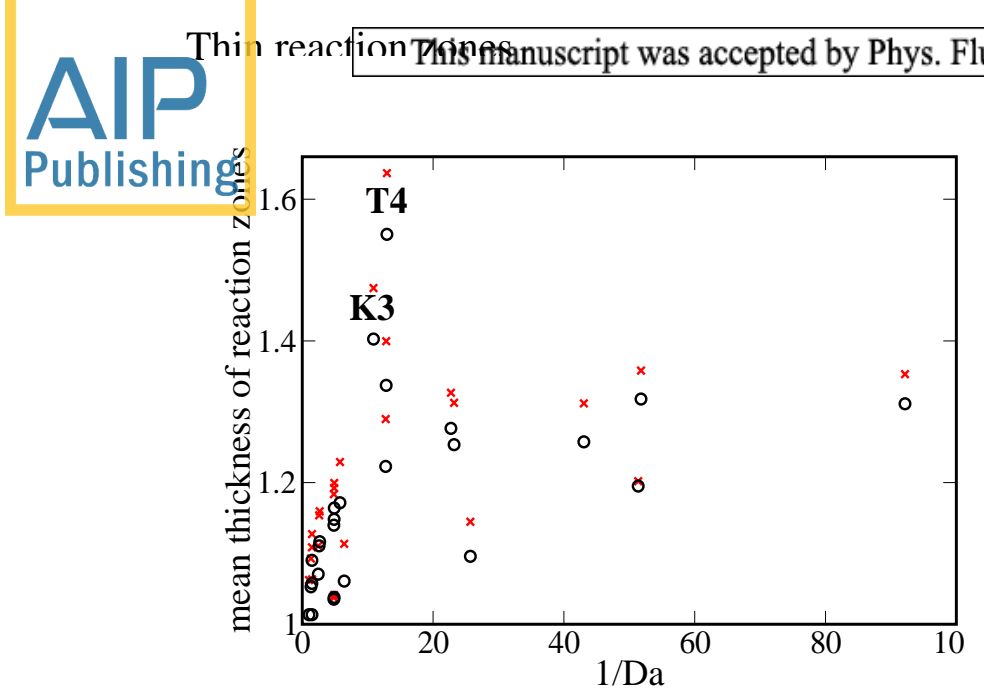

(a)

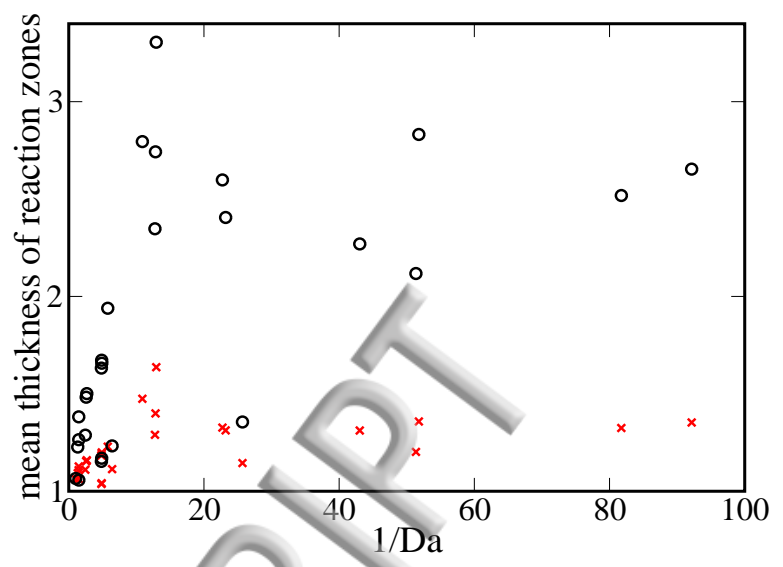

(b)

FIG. 5. (a) Mean values of normalized reaction-zone thickness evaluated using Eq. (15) with $c_{r, 1}<c<c_{r, 2}$ (crosses) or $c_{w}-0.005<c<c_{w}+0.005$ (circles). (b) Comparison of normalized reaction-zone thicknesses computed using Eq. (15) (crosses) and Eq. (16) (circles).

with $S c R e_{t}$, but the data point are widely scattered. The same data on $\bar{u}_{c} / S_{L}$ plotted vs. $u^{\prime} / S_{L}$ show a more clear trend, see Fig. 4(b).

It is also worth noting the following point. A comparison of calculated $\langle\delta A\rangle_{r}$ or $U_{T} / S_{L}$ between waves D4 and $\mathrm{H} 4$ or $\mathrm{H} 1$ and $\mathrm{H} 2$ shows a very weak influence of $\mathrm{Ze}$ on these key characteristics of turbulent reaction waves. Indeed, $\langle\delta A\rangle_{r}=5.71$ and 6.0 in cases D4 $(Z e=6.0)$ and $\mathrm{H} 4(\mathrm{Ze}=17.1)$, respectively, or 3.65 and 3.91 in cases $\mathrm{H} 1(\mathrm{Ze}=6.0)$ and $\mathrm{H} 2(\mathrm{Ze}=17.1)$, respectively. While some increase in $\langle\delta A\rangle_{r}$ with $Z e$ is observed, the effect is weak. Similarly, $U_{T} / S_{L}=7.59$ and 7.37 in cases D4 and $\mathrm{H} 4(\mathrm{Da}=0.02)$, respectively, or 4.09 and 4.07 in cases $\mathrm{H} 1$ and $\mathrm{H} 2(D a=0.21)$, respectively, with the waves in each pair being characterized by the same values of $u^{\prime} / S_{L}, L_{11} / \delta_{L}$, $R e_{t}, S c, D a$, and $K a$, see Table II. Weak influence of $Z e$ on $U_{T}$ was also observed in a recent DNS study of highly turbulent, single-step chemistry, premixed flames ${ }^{60}$. These results are explained in Section IH.

\section{Reaction zone thickness}

Figure 5 reports normalized mean values of local reaction-zone thickness, which was evaluated using either of the equations

$$
\left\langle\boldsymbol{\delta}_{r}\right\rangle_{\Sigma, c_{1}<c<c_{2}}=\frac{\langle\Sigma /|\nabla c|\rangle_{c_{1}<c<c_{2}}}{\langle\Sigma\rangle_{c_{1}<c<c_{2}}}=\frac{1}{\langle|\nabla c|\rangle_{c_{1}<c<c_{2}}}
$$




$$
\left\langle\boldsymbol{\delta}_{r}\right\rangle_{c_{1}<c<c_{2}}=\left\langle\frac{1}{|\nabla c|}\right\rangle_{c_{1}<c<c_{2}}
$$

and normalized to the thickness obtained by averaging either $|\nabla c|$ or $1 /|\nabla c|$, respectively, over $c_{1}<c<c_{2}$ in the corresponding 1D laminar reaction wave. Here, $\Sigma \equiv|\nabla c|$ is the reaction-wavesurface density and $c_{k}=c_{r, k}$, see crosses in Fig. 5(a) and all symbols in Fig. 5(b), or $c_{1}=$ $c_{w}-0.005$ and $c_{2}=c_{w}+0.005$, see circles in Fig. 5(a). Equation (15) gives the thickness weighted by $\Sigma$. In particular, such a type of averaging is widely used in premixed turbulent combustion ${ }^{25}$. Equation (16) defines the thickness averaged over the reaction zone volume.

Figure 5(a) shows that the former normalized thickness is quite close to unity even at $D a$ as low as 0.01 and $K a$ as high as 587. The normalized mean thickness $\left\langle\delta_{r}\right\rangle_{\Sigma, c_{1}<c<c_{2}}$ is smaller than 1.4 in all cases with the exception of waves $\mathrm{K} 3$ and $\mathrm{T} 4$, which are characterized by the highest ratios $S c R e_{t}$ of turbulent and laminar diffusivities. Even in these two cases, the thickness is smaller than 1.6, i.e., reaction-zone broadening is weakly pronounced after averaging.

For the thickness $\left\langle\boldsymbol{\delta}_{r}\right\rangle_{c_{r, 1}<c<c_{r, 2}}$, which is averaged without weighting by $\Sigma$, the broadening effect is more pronounced, cf. circles and crosses in Fig. 5(b). Nevertheless, when $D a<0.1$, no consistent variation in the normalized thickness $\left\langle\boldsymbol{\delta}_{r}\right\rangle_{c_{r, 1}<c<c_{r, 2}}$ with decreasing $D a$ is observed and the thickness values are scattered between 2.1 and 2.8 with the exception of two cases. The largest normalized $\left\langle\boldsymbol{\delta}_{r}\right\rangle_{\Sigma, c_{r, 1}<c<c_{r, 2}}$ and $\left\langle\boldsymbol{\delta}_{r}\right\rangle_{c_{r, 1}<c<c_{r, 2}}$, respectively 1.6 and 3.3, were computed for case T4, see also slices in Fig. 2(a). Note the substantial difference between these thickness values; i.e., the ratio $\left\langle\boldsymbol{\delta}_{r}\right\rangle_{c_{r, 1}<c<c_{r, 2}} /\left\langle\boldsymbol{\delta}_{r}\right\rangle_{\Sigma, c_{r, 1}<c<c_{r, 2}}$ is close to two when $D a<0.1$. This can be attributed to the existence of regions where the reaction rate $W(c)$ is high while the gradient $|\nabla c|$ is low due to an overlap between reaction zones. High local values of $1 /|\nabla c|$ imply large values of thickness given by Eq. (16), and the probability of finding such regions apparently increases with decreasing $D a$.

Figure 6(a) Shows that PDFs of the normalized local thickness $|\nabla c|_{w, L} /|\nabla c|_{w}$ peak near unity at various $D a$, including $D a \ll 1$. In Fig. 6(b), the probability of finding $|\nabla c|_{w, L} /|\nabla c|_{w}>2$ increases with decreasing $D a$, which implies a more frequent occurrence of reaction zones broadened by small-scale turbulent eddies. At the same time, there is a substantial probability of finding narrowed reaction zones, where $|\nabla c|_{w, L} /|\nabla c|_{r}<1$ due to the local turbulent stretching. The former probability is close to $50 \%$ in the few cases (D3, D4, D9) characterized by the lowest $D a$, while the latter is about $20 \%$ in these extreme cases. In most other cases such as T2 and B15, the former and latter probabilities are significantly smaller than $50 \%$ and larger than $20 \%$, respectively.

An increase in Ze reduces the probability of $|\nabla c|_{w, L} /|\nabla c|_{w}>2$, cf. cases D4 and $\mathrm{H} 4$, but this 


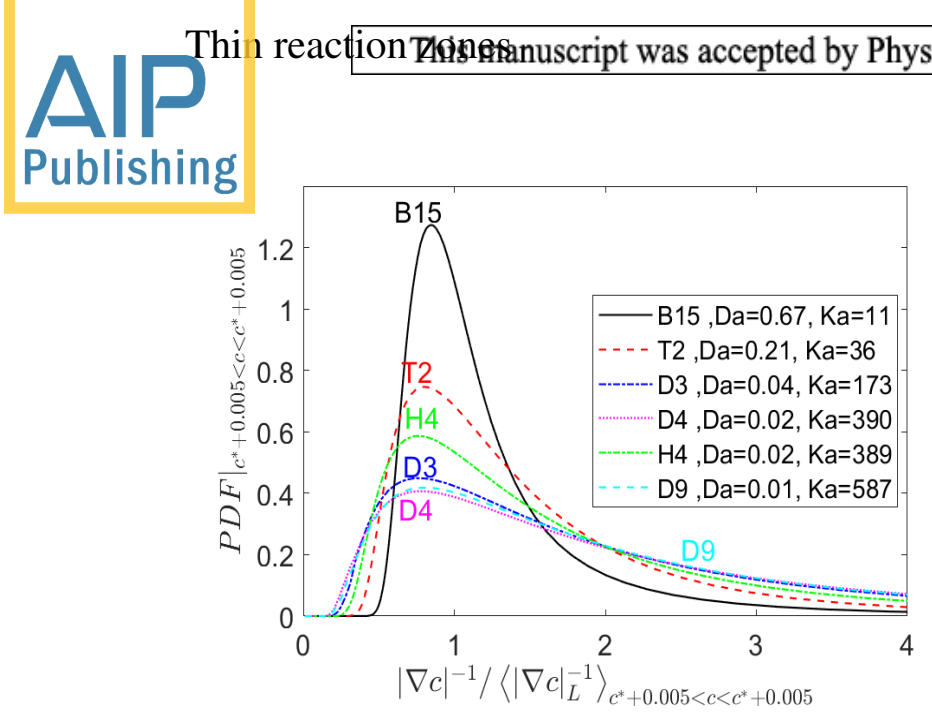

(a)

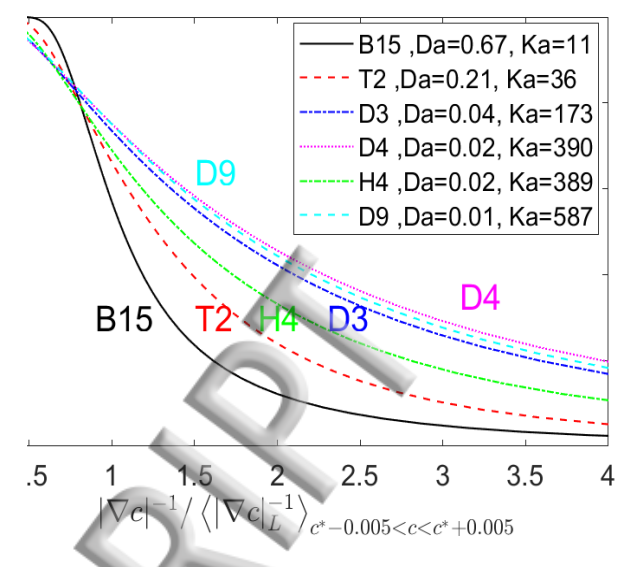

(b)

FIG. 6. (a) Probability density functions and (b) cumulative probabilities for the normalized local reactionzone thickness $|\nabla c|_{w, L} /|\nabla c|_{w}$ conditioned to $c_{w}-0.005<c<c_{w}+0.005$.

effect is also moderate.

All in all, the present DNS results demonstrate that reaction-zone broadening is weak or moderate in the statistical sense, i.e. both $\left\langle\delta_{r}\right\rangle_{c_{r, 1}<c<c_{r, 2}}$ and $\left\langle\delta_{r}\right\rangle_{\Sigma, c_{r, 1}<c<c_{r, 2}}$ are sufficiently close to the counterpart measures of the laminar wave thickness, but are much less than the mean wave brush thickness $\delta_{T}$ discussed in Section II B 4.

\section{Mean reaction rate}

It is of interest to note that, even though the PDFs plotted in Fig. 6 indicate a significant probability of occurrence of thin reaction zones characterized by values of $|\nabla c|^{-1}$ lower than the corresponding laminar values, the PDFs of $c$ presented in Fig. 7(b) show that non-negligible probabilities of finding various values of $c(\boldsymbol{x}, t)$ fall within a relatively narrow interval of $c$, shifting from $c=0$ to $c=1$ with increasing $\bar{c}(x)$. The behavior of the PDFs implies weak fluctuations of $W(c)$ and is consistent with the key assumption made by Damköhler ${ }^{9}$ that the influence of turbulence on the mean reaction rate $\overline{W(c)}$ is negligible compared to mixing enhancement by small-scale turbulent eddies. This feature of $P(c)$ is well pronounced in case D9, characterized by a very low $D a=0.01$, see Fig. 7(b), but is not pronounced in case T2, characterized by a larger $D a=0.21$, see Fig. 7(a). Moreover, the PDFs computed in case D9 show that the probability of finding values of $c$, for which reaction rate $W(c)$ does not vanish, is negligible when $\bar{c}=0.1$ and 0.3 , and is very low when $\bar{c}=0.5$. Accordingly, the mean reaction rate $\bar{W}$ should be non-negligible 


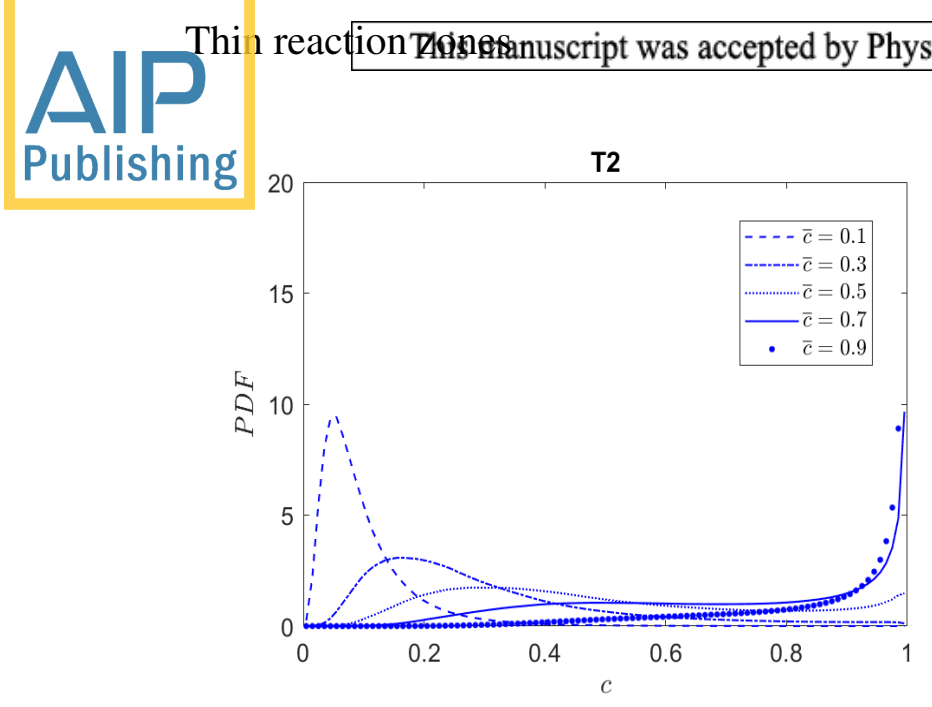

(a)

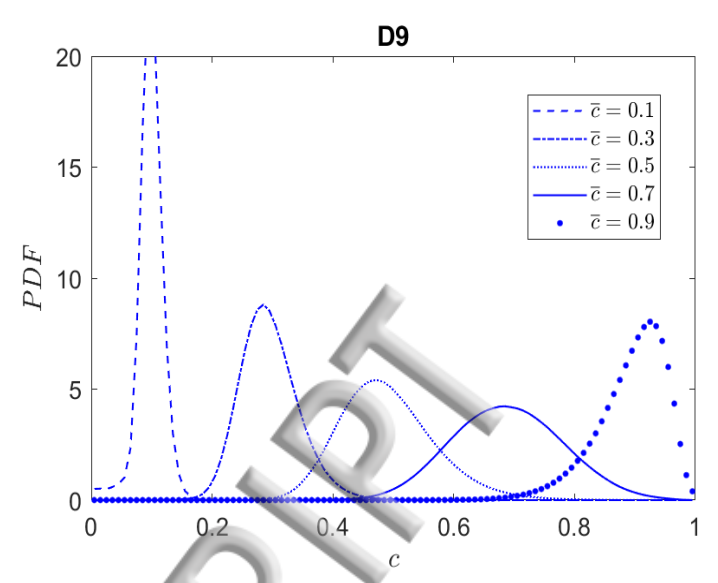

(b)

FIG. 7. Probability density functions of $c$ obtained at different $\bar{c}(x)$ specified in legends. (a) Case T2, $D a=0.21$. (b) Case D9, $D a=0.01$.

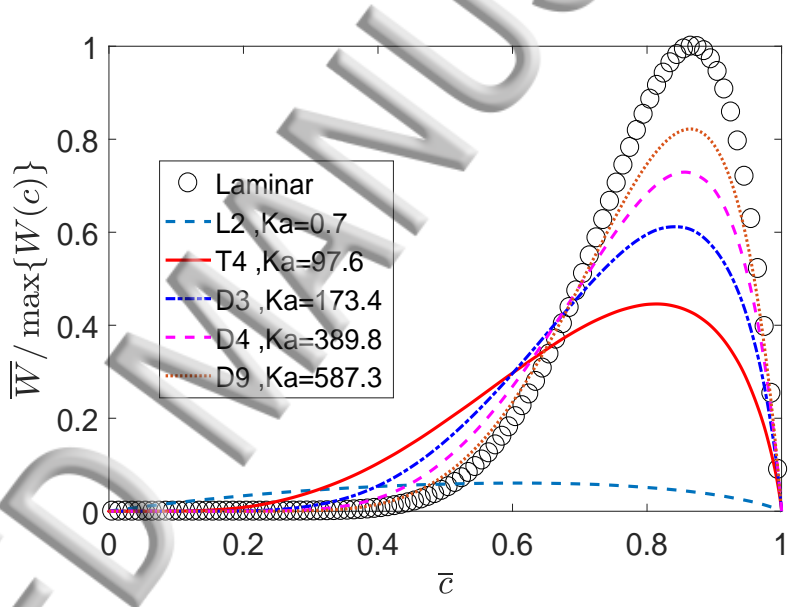

FIG. 8. Normalized instantaneous and mean reaction rates, $W / \max \{W(c)\}$ (symbols) and $\bar{W} / \max \{W(c)\}$ (curves), vs. instantaneous and mean reaction progress variables, $c$ and $\bar{c}$, respectively. Cases are specified in the legend. For comparison, results simulated in case L2 $\left(u^{\prime} / S_{L}=1, L_{11} / \delta_{L}=12.4, \operatorname{Re}_{t}=158, K a=0.72\right)$, which is representative for the flamelet combustion regime, are plotted in cyan dashed line.

only when $\bar{c}>0.5$. Such variation of $\bar{W}$ with $\bar{c}$ is similar to that of $W(c)$ in laminar reaction waves but is significantly different from the variation of $\bar{W}$ with $\bar{c}$ in reaction waves characterized by $D a>1$.

Indeed, Fig. 8 shows that the graph of computed mean reaction rate $\bar{W}$ plotted against mean reaction progress variable $\bar{c}$, see curves, changes shape significantly with decreasing $D a$. When $D a<1$, it has a very different shape from that of $\bar{W}(\bar{c})$ in case L2, which is representative for the 


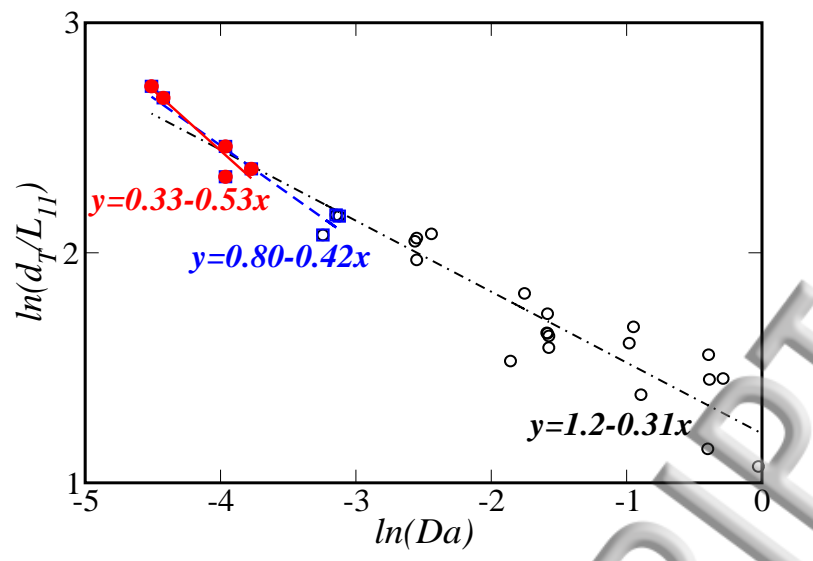

FIG. 9. Mean wave brush thickness $\delta_{T} / L_{11}$ vs. Damköhler number. Black open circles, blue open squares, and red filled circles show data obtained from all 26 cases characterized by $D a<1$, eight cases characterized by $D a \leq 0.044$, and five cases characterized by $D a \leq 0.023$, respectively. Data points are fitted with black dotted-dashed, blue dashed, and red solid lines, respectively.

flamelet combustion regime $(D a=12.4)$. Moreover, $\bar{W}(\bar{c})$ approaches the laminar profile $W(c)$, see symbols, as Da decreases. This trend is fully consistent with Damköhler's hypothesis.

The trend implies that modeling of highly turbulent reacting flows can be simplified; i.e., the influence of turbulent fluctuations on the mean reaction rate $\bar{W}$ can be neglected at sufficiently high $K a$ (low $\mathrm{Da}$ ). Indeed, Duwig et al. ${ }^{1}$ reported reasonable agreement between the results of their large eddy simulations performed by discarding the subfilter fluctuations in $W$ and experimental data obtained by Dunn et al. ${ }^{43}$ from a highly turbulent piloted flame.

\section{Mean wave brush thickness}

Figure 9 shows DNS results (black symbols) on the mean wave brush thickness

$$
\delta_{T}=\frac{1}{\max \left\{\left|\nabla_{x} \bar{c}\right|(x)\right\}},
$$

with dotted-dashed black line fitting the data points with $\delta_{T} / L_{11} \propto D a^{-0.31}$. It is worth remembering that the integral length scale $L_{11}$ is evaluated by integrating the auto-correlation function $R_{11}^{u}(r)$, see Section II A 3 , and ratios of $L_{11} / \delta_{L}$ reported in the next-to-the-right column in Table II vary from 0.39 to 8.27 . Other symbols and lines are discussed in Section II C 2. Similarly to $U_{T} / u^{\prime}$, the ratio $\delta_{T} / L_{11}$ decreases with increasing $D a$, but the data are more scattered compared to the DNS data on $U_{T} / u^{\prime}$ vs. $D a$ in Fig. 3(a). 


\section{Relevance of constant-density single-reaction waves to combustion}

The present DNS results agree qualitatively with available experimental and DNS data obtained from premixed turbulent flames. For instance, Eq. (4) was validated by combustion experiments ${ }^{32}$. Moreover, Eq. (4) was also validated by DNS data ${ }^{8}$ obtained from thermonuclear turbulent flames characterized by moderate density ratios $\sigma<2 ; K a=230$; and $D a=0.006,0.025$, and 0.1 . It is worth noting that DNS data computed at $D a=0.4$, see Fig. 3 in Ref. 8 , showed some deviations from Eq. (4). Furthermore, Eq. (4) was recently validated by complex-chemistry DNS data obtained from highly turbulent lean methane-air and hydrogen-air flames, see Fig. 4 in Ref. 34. It is of interest to note that, in those DNSs, Eq. (4) was validated even under conditions where the concept of DRZ was not expected to apply and this finding was considered "unclear", see p. 6 in Ref. 34 .

Moreover, the present DNS results plotted in Fig. 3(b) are fully consistent with DNS data obtained recently by Nivarti and $\mathrm{Cant}^{62}$ from five different premixed turbulent flames characterized by $\sigma=7.33, D a>0.5, K a<50, u^{\prime} / S_{L}<30\left(u^{\prime} / S_{L} \leq 18\right.$ at the leading edge of the simulated mean flame brushes), and a single value of $L / \delta_{L}$. Those data show that an increase in $U_{T} / S_{L}$ by $u^{\prime}$ is mainly controlled by an increase in the flame-surface area by $u^{\prime}$, see Fig. 7 in Ref. 62. The present DNS data indicate the/same trend, but in the constant-density case and in significantly wider ranges of $0.01 \leq D a<1,6.5<K a<590$, and $2 \leq u^{\prime} / S_{L} \leq 90$. Note that in experimental and DNS papers cited in the present subsection, different definitions of the laminar flame thickness $\delta_{L}$ were adapted, but all of them yielded $\delta_{L}$ significantly larger than $\delta_{L}=D_{u} / S_{L}$ used in the present work. Here, subscript $u$ designates unburned reactants. Accordingly, the values of Damköhler and Karloyitz numbers reported in those papers are significantly lower or higher, respectively, than the values of $D a$ or $K a$, respectively, re-calculated using Eqs. (2) and (3), respectively. Reported in the present subsection are the latter (re-calculated) values of $D a$ or $K a$.

Results of complex-chemistry DNSs performed for various paraffin fuels (methane, toluene, $n$-heptane, and iso-octane) also show that the ratio $U_{T} / S_{L}$ is mainly controlled by the area of reaction-zone surface at $K a$ as large as 260, see Figs. 5-7 in Ref. 63.

The fact that turbulent consumption velocities simulated in the case of a constant density and single-step chemistry agree well with experimental and DNS data obtained from premixed tur- 
Thin reaction Tongfanuscript was accepted by Phys. Fluids. Click here to see the version of record.

Publishibugent flames characterized by Lewis numbers $L e \approx 1$ is not surprising. Indeed, first, turbulent burning velocities evaluated using single-step and multi-step chemistry were recently compared in two independent DNS studies ${ }^{63,64}$. Obtained results show that "mean turbulent flame properties such as burning velocity and fuel consumption can be predicted with the knowledge of only a few global laminar flame properties", see p. 294 in Ref. 63, and "the global mechanism is adequate for predicting flame speed“, see p. 53 in Ref. 64. Moreover, target-directed experiments ${ }^{65}$ performed using the well-recognized Leeds fan-stirred bomb facility do not show a notable effect of combustion chemistry on turbulent flame speed either. Such effects are commonly expected to be of substantial importance when local combustion extinction occurs, but this phenomenon is beyond the scope of the present study.

Second, (i) the vast majority of approximations of experimental data on turbulent flame speed $^{24,59}$ do not invoke the density ratio $\sigma$, thus, implying a weak influence of $\sigma$ on $S_{T}$, (ii) recent target-directed experiments ${ }^{66}$, as well as earlier measurements ${ }^{65}$, did not reveal a substantial influence of $\sigma$ on $S_{T}$, and (iii) recent DNS studies, e.g., see Figs. 10 and 11 in Ref. 18 or Fig. 2a in Ref. 67, do not indicate such an influence either.

As far as the reaction zone thickness is concerned, Figs. 5 and 6 also agree qualitatively with DNS and experimental data obtained from turbulent premixed flames. For instance, Driscoll ${ }^{68}$ thoroughly reviewed experimental data available a decade ago, but did not find any clear evidence of existence of distributed reaction zones in premixed turbulent flames. More recently, Chowdhury and Cetegen ${ }^{69}$ analyzed experimental data obtained from highly turbulent flames stabilized using a bluff body and concluded that there was "no evidence of broadly distributed heat release zones", see p. 320 in Ref. 69. In other recent experimental studies ${ }^{31,36,42}$ of flames characterized by high (low) values of $\mathrm{Ka}(\mathrm{Da})$, reaction-zone broadening was observed, but the effect was moderate. In particular, the ratio of a mean thickness of a reaction zone in a turbulent flow to the corresponding thickness of the laminar reaction zone was less than 2.0, see Fig. 11 in Ref. 31, or 3.0, see Fig. 8 in Ref. 42, at Karlovitz numbers as high as 300 or 135, respectively.

Statistically significant broadening of reaction zones was not documented in earlier DNS studies of premixed turbulent flames with single-step ${ }^{70-72}$ or complex ${ }^{73-79}$ chemistry. Thévenin $^{73}$ did not observe a significant influence of turbulence on reaction zone thickness at moderately low (high) $D a(K a)$, see p. 635 in Ref. 73. Figures 7 and 8 in Ref. 70, Figs. 4, 6, and 7 in Ref. 74, and Fig. 6 in Ref. 76 do not show such an effect either. Figures 7 and 8 in Ref. 71 and Fig. 12 in Ref. 72 indicate some reaction-zone broadening, but the effect is weak. Figure 3 in Ref. 77 
Thin reaction Zqgnefanuscript was accepted by Phys. Fluids. Click here to see the version of record.

Publishishgovs statistical thinning of reaction zones with decreasing $D a$, but this effect appears to result from Lewis number and preferential diffusion phenomena discussed elsewhere ${ }^{4,13}$. Figure 8 in Ref. 78 also indicates statistical thinning of reaction zones with increasing $K a$ from 13 to 700 , but these data were computed during an early stage of flame development and the behavior of the mean reaction-zone thickness was subject to transient effects.

Figure 13 in Ref. 75 shows an increase in the normalized mean thickness of reaction zone from unity to 2.6, with the thickness being calculated as "the local distance around the peak source term where its value" was "above $5 \%$ of the laminar value". However, the choice of the 5\%-boundary appears to overestimate the reaction-zone thickness and the magnitude of the broadening effect. For instance, even in the case characterized by the highest $K a$ and the aforementioned normalized mean thickness equal to 2.6 , only $20 \%$ of fuel consumption occurred outside reaction layers whose thickness was equal to the laminar reaction-zone thickness, see p. 3350 in the discussed paper. In other words, fuel consumption was mainly localized to thin zones in those simulations.

Figures 12 and 14b in Ref. 79 report dependencies of the ratio $\langle|\nabla c|\rangle_{c} /|\nabla c|_{c, L}$ of conditionally averaged gradients $|\nabla c|$ on the value of $c$ that the gradients were conditioned to. Those data were obtained from a highly turbulent methane-air jet flame. At $c=0.8$ associated with the peak heat release rate, this ratio was larger than 0.5 , thus indicating statistically moderate broadening of the reaction zone, with the effect magnitude being comparable with the present DNS data obtained in case T4, see Fig. 6a.

Figure 5a in Ref. 34 did not show statistically substantial broadening of reaction zones at $K a$ as high as about 3650 for lean methane-air flames. However, results obtained from lean hydrogen-air flames characterized by a comparable $K a$ were different. On the one hand, 2D images reported in Figs. 2 and 3 in the discussed paper appear to show significant ("by around two orders of magnitude") broadening of heat-release zones. On the other hand, temperature gradient conditioned to these zones indicates statistically moderate (about $50 \%$ ) broadening of them. It is also worth remembering that lean hydrogen-air mixtures are poorly suited for differing the influence of small-scale turbulence on preheat and reaction zones, because the thicknesses of the two zones are comparable in the laminar flames of such mixtures.

Finally, while 2D images reported by Aspden et al. ${ }^{7,80}$ also indicate reaction-zone broadening at high $\mathrm{Ka}$ in thermonuclear and lean hydrogen-air flames, respectively, the magnitude of the effect was not statistically quantified and contribution of inclined (to the 2D slices) zones to their apparent broadening was not investigated in the cited papers. Moreover, the thermonuclear flames 
Thin reaction Zqugnanuscript was accepted by Phys. Fluids. Click here to see the version of record.

Publishiwgre subject to strong Lewis number effects.

As far as experimental or DNS data on fully-developed mean flame brush thickness or the difference between $\bar{W}(\bar{c})$ and $W(\bar{c})$ are concerned, the present authors are not aware of such data obtained from highly turbulent flames.

Thus, the above comparison of the present DNS data with experimental and DNS data obtained from premixed turbulent flames shows encouraging agreement for the major trends (scaling of turbulent consumption velocity, which is mainly controlled by an increase in reaction-zone-surface area, and statistically moderate broadening of reaction zones), thus, implying relevance of results of research into a simple problem of propagation of a single-reaction wave in constant-density turbulence to turbulent burning. Therefore, in spite of the simplicity of the simulated problem, it does offer an opportunity to catch certain governing physical mechanisms of premixed turbulent combustion. Accordingly, research into the problem may provide better insight into fundamentals of the influence of turbulence on premixed flames. Nevertheless, when applying the present results or results obtained in other (e.g., future) studies of the simple problem to turbulent burning, other important physical mechanisms associated with preferential diffusion, thermal expansion, and complex chemistry should be borne in mind.

In this regard, two issues are worth emphasizing. First, on the one hand, there is a widely accepted hypothesis that thermal expansion effects may impede reaction-zone broadening by smallscale turbulent eddies, because such eddies dissipate in preheat flame zones due to dilatation and increased viscous dissipation $34,36,38,68$. This hypothesis is based on results of numerous studies of effects of a single yortex or vortex pair on a laminar premixed flame. Numerical and experimental research into that problem was pioneered by Poinsot et al. ${ }^{23}$ and Roberts et al. ${ }^{81}$, respectively. Results obtained in subsequent studies are reviewed elsewhere ${ }^{82,83}$. If the hypothesis is accepted, then, an "efficient" $K a$ experienced by constant-density reaction zones appears to be significantly higher than an "efficient" $K a$ experienced by similar but variable-density reaction zones in statistically the same incoming flow of reactants. Indeed, dilatation vanishes in the former case and the rate of viscous dissipation is not increased due to the reaction if $\rho=$ const and $v=$ const. Accordingly, the critical value $K a^{*}$ associated with significant broadening of reaction zones should be lower in the case of a constant density.

On the other hand, baroclinic torque can overwhelm the influence of dilatation and viscous dissipation and can increase vorticity in the reaction zone in a flame ${ }^{84}$. Moreover, in intense Kolmogorov turbulence, the flux of energy from large to small eddies can allow the latter eddies 
Thin reaction Zq9nefanuscript was accepted by Phys. Fluids. Click here to see the version of record.

$A \mid P^{\text {m }}$

Publishitigg urvive and to penetrate into reaction zones.

Definitely, the issue requires further research. Nevertheless, we may note that (i) if statistically significant broadening of reaction zones is not observed in constant-density turbulent flows addressed in the present DNS, then, such a result is unlikely to be reversed due to thermal expansion in flames and (ii) the upper boundary $K a=K a^{*}$ of the Thin Reaction Zone (TRZ) regime discussed in the next subsection is unlikely to decrease with increasing the density ratio. For instance, DNS data ${ }^{71,85,86}$ indicate that the influence of combustion-induced thermal expansion on turbulence decreases with increasing $u^{\prime} / S_{L}$ and reduces to an increase in the Kolmogorov scales in burned gas if $u^{\prime} / S_{L}$ is sufficiently large.

Second, in the simple case studied in the present work, the reaction surface cannot locally be broken, i.e., it does not have holes ${ }^{2}$. On the contrary, due to heat losses, preferential diffusion and complex chemistry effects, local or global combustion quenching can occur in highly turbulent premixed flames and, consequently, heat-release zones can locally be broken. However, available data on conditions required for local or global quenching are contradictory.

On the one hand, experiments with expanding statistically spherical flames ${ }^{87-90}$ clearly showed combustion extinction at moderately high Karlovitz numbers, see also recent experimental data obtained by Chowdhury \& Cetegen ${ }^{69}$, Kariuki et al. ${ }^{91}$, and Zhou et al. ${ }^{92}$ from other flame configurations. Moreover, local combustion extinction was also detected in a recent DNS study ${ }^{75}$ but "only in non-unity Lewis number simulations" (p. 3341), with the probability of the extinction decreasing at higher $K a$.

On the other hand, if burning is well supported by hot combustion products and the flame is shielded from cool air, then, local combustion extinction seems to be of minor importance at least at Karlovitz numbers reached in recent experiments by Driscoll's group ${ }^{31,35-37}$. In particular, these authors clearly stated that (i) the studied six cases with high $K a$ did "not exhibit a substantial degree of localized extinction", while rare events of local extinction resulted from "cool gas entrainment", see p. 407 in Ref. 31, (ii) "broken reactions ... were not observed for any of the six cases, even for extreme turbulence levels in the reactants of $u^{\prime} / S_{L}=243$ “, see p. 1816 in Ref. 36, (iii) extinction events were "rare", see p. 4597 in Ref. 37, and any local extinction was "insignificant", see p. 1808 in Ref. 35. In other very recent set of experiments with jet flames ${ }^{39-42}$ characterized by $K a \gg 1$, local combustion extinction was observed, but only far from the nozzle, with this effect being attributed to cool air entrainment. DNS studies ${ }^{80,93}$ of lean hydrogen-air flames characterized by $K a \gg 1$ did not reveal any local extinction either. 
Publishing Thus, regimes of highly turbulent burning characterized by the same $K a$ and the same $D a$ seem to be different depending on flame configuration and stabilization method. Accordingly, results of the present DNSs appear to be relevant to highly turbulent flames supported by hot combustion products, but not relevant to other, e.g., expanding statistically spherical, flames, where extinction phenomena due to heat losses, preferential diffusion and complex chemistry effects play a crucial role.

\section{Regimes of the influence of intense turbulence on reaction waves}

As far as Damköhler's classical theory is concerned, on the one hand, certain present DNS data are consistent with it. First, the data very well-support Eq. (4), which results from the theory. Second, Fig. 8 shows a clear trend of $\overline{W(c)}$ to $W(\bar{c})$ with decreasing $D a$, in line with the theory. Third, the computed decrease in $\delta_{T} / L_{11}$ with increasing $D a$ is qualitatively consistent with the theory. Indeed, if following Damköhler ${ }^{9}$, the influence of turbulence on a reaction wave is solely reduced to mixing enhancement, then $\delta_{T} / \delta_{L}$ should be proportional to $\sqrt{D_{T} / D} \propto \sqrt{S c R e_{t}}$. Consequently,

$$
\frac{\delta_{T}}{\mathrm{~L}_{11}} \propto D a^{-1 / 2},
$$

where $D_{T} \propto u^{\prime} L$ is turbulent diffusivity. Fitting dotted-dashed line in Fig. 9 shows a similar dependence, but it is less pronounced (the power exponent is -0.31).

Figures 8 and 9, considered together, imply that, probably, lower values of $D a$ (or/and higher values of $K a$ ) are required in order for $\overline{W(c)}$ to reach $W(\bar{c})$ and Eq. (18) to hold. For instance, even at $D a$ as low as 0.01 (case D9), there are differences between $\overline{W(c)}$ and $W(c)$ in Fig. 8. To assess the above assumption, the DNS data on $\delta_{T}$ obtained (i) from eight waves characterized by $D a \leq$ 0.044 and (ii) from five waves characterized by $D a \leq 0.023$ were separately processed. Results shown in (i) open blue squares and dashed line and (ii) red filled circles and solid line, respectively, in Fig. 9 do indicate that the fitting power exponent $p$ is sufficiently close to -0.5 when $D a$ is low. Therefore, the DNS data plotted in Fig. 9 do not seem to contradict to Damköhler's theory.

On the other hand, certain DNS data appear to contradict to the DRZ concept. First, Fig. 5 does not indicate statistically substantial broadening of reaction zones. Second, and the most important, comparison of circles and squares in Fig. 3(b) clearly shows that an increase in $U_{T} / S_{L}$ is mainly controlled by an increase in the reaction-surface area and this result contradicts directly to the concept. Probably, substantially lower (higher) values of $D a(K a)$ are required in order to 
Publishidegfect mixing enhancement in broadened reaction zones as the primary mechanism of an increase in $U_{T} / S_{L}$.

At first glance, the present DNS data appear to be fully consistent with the TRZ concept of highly turbulent premixed combustion, which was put forward by Peters ${ }^{3}$ by considering the simple problem investigated by us (constant density, single-step chemistry, etc.). At the same time, the present DNS data indicate that the boundary $K a=K a^{*}$ of this regime should be associated with values of $K a^{*}$ significantly higher, e.g., $K a=587$ in case D9, than the simple criterion $K a^{*}=100$ suggested by Peters ${ }^{3}$. Furthermore, it is worth remembering that Peters attributed "an upper limit for the" TRZ regime to penetration of Kolmogorov eddies into reaction zones, see p. 122 in Ref. 3. Subsequently, Peters obtained the criterion $K \mathrm{~K}^{*}=100$ by assuming that the laminar reaction-zone thickness $\delta_{r}=0.1 \delta_{L}$. However, under conditions of the present constant-density DNS, difference in $\delta_{r}$ and $\delta_{L}$ is much less ${ }^{47}$, e.g., $\delta_{r}=0.72 \delta_{L}$ if $Z_{e}=6.0$. Consequently, $\eta_{K}<\delta_{r}$ if $K a \geq 2$, i.e., in all 23 cases characterized by $Z e=6.0$ and $D a<1$, with $\delta_{r} / \eta_{K}$ being larger than 17 in case D9. Therefore, if Peters' arguments are directly applied to the present DNS data, then, the simulated waves are not associated with the TRZ regime.

Peters $^{3}$ not only introduced the TRZ regime, but also modeled it and, in particular, obtained the following equations

$$
\begin{gathered}
\frac{U_{T}}{u^{\prime}}=b_{1} \frac{S_{L}}{u^{\prime}}-0.195 D a+\left[(0.195 D a)^{2}+0.78 D a\right]^{1 / 2} \\
\delta_{T}=b_{2} L
\end{gathered}
$$

where, $b_{1}=1.0$ and $b_{2}=1.78$.

Black circles in Fig. 10 show that the original Eq. (19) with $b_{1}=1$ agrees with the DNS data reasonably well, but the agreement is much better if $b_{1}=0$, see red crosses. This is not surprising, because, when $D a \rightarrow 0$, Eq. (19) with $b_{1}=0$ reduces to Damköhler's classical scaling given by Eq. (4), e.g., difference between the values of $U_{T} / u^{\prime}$ calculated using Eq. (19) with $b_{1}=0$ and its low-Da limit $\left(U_{T} / u^{\prime}=\sqrt{0.78 D a}\right)$ is as low as $10 \%$ when $D a=0.19$.

However, DNS data plotted in Fig. 9 contradict to Eq. (20), which was directly used by Peters $^{3}$ to arrive at Eq. (19). Moreover, Eq. (20) is contradicted by experimental data discussed elsewhere $^{4,24}$ and by recent DNS data ${ }^{46}$. Consequently, even if a single Fig. 10 appears to well support the model by Peters ${ }^{3}$, the opposite conclusion should be drawn when assessing the model by considering Figs. 9 and 10 jointly. The model in itself is critically discussed elsewhere ${ }^{4,94-96}$. 
Thin reaction Tonghanuscript was accepted by Phys. Fluids. Click here to see the version of record.

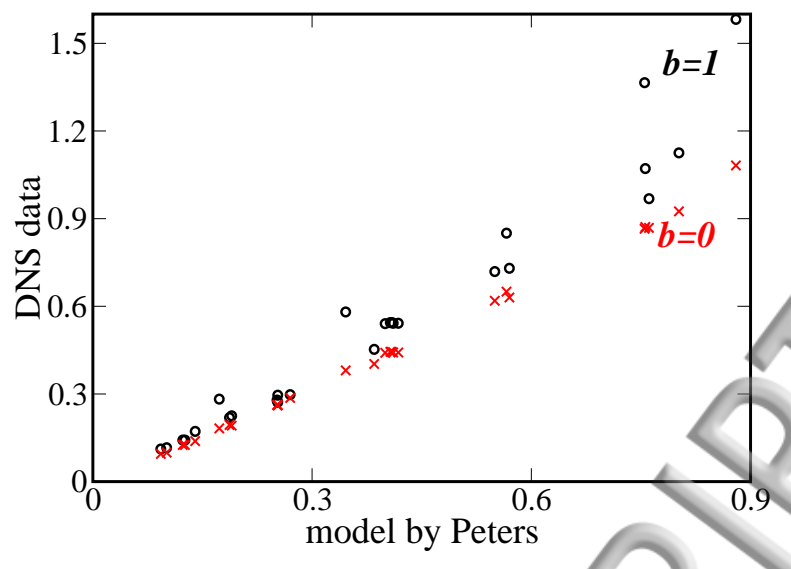

FIG. 10. Comparison of the values of $U_{T} / u^{\prime}$ extracted from the DNS data (ordinate axis) with the values of $U_{T} / u^{\prime}$ calculated (abscissa axis) using Eq. (19) with $b_{1}=1$ (black circles) or $b_{1}=0$ (red crosses).

\section{INFINITELY THIN REACTION ZONES IN INTENSE TURBULENCE: A THEORY}

As discussed above, Peters' theory is contradicted by certain present DNS data. Therefore, the data call for development of another theory of propagation of a thin reaction zone in intense turbulence. This is the goal of the present section.

\section{A. Statement of the problem}

Let us consider a statistically planar, 1D, reaction wave that propagates from right to left along $x$-axis in homogeneous isotropic turbulence, but does not affect it, because the reaction affects neither the density $\rho$ nor the viscosity $v$. We address the case of a single-step chemistry, $L e=1$, and intense turbulence characterized by a high $\operatorname{Re}_{t} \gg 1$, a low $D a<1$ and, hence, a high $K a \gg 1$.

To explore a physical scenario opposite to the widely accepted paradigm of distributed reactions, let us study a reaction whose rate $W$ depends on $c$ in the extremely nonlinear manner, i.e., $W[c(\boldsymbol{x}, t)]$ vanishes outside a very thin reaction zone, whose thickness $\delta_{r}$ is much less than both the Kolmogorov length scale $\eta_{K}$ and the laminar wave thickness $\delta_{L}$. A similar limiting case is often analyzed in theoretical studies of laminar premixed flames ${ }^{97,98}$ following the pioneering ideas by Zel'dovich and Frank-Kamenetskii who developed the well-known ZFK theory of laminar premixed flames ${ }^{99}$ by seeking for a solution to governing transport equations for large $\mathrm{Ze}$ in Eq. (8). In the considered limiting case, the reaction zone degenerates to a reaction surface $c\left(\boldsymbol{x}_{r}, t\right)=1$, 
Publishiwgich separates the mixture of reactants and products, where $0 \leq c(\boldsymbol{x}, t)<1$, from the equilibrium products, where $c(\boldsymbol{x}, t)=1$ and $\boldsymbol{x} \neq \boldsymbol{x}_{r}$. In the following, the mixture of the reactants and the products is called reactants for brevity, i.e., $0 \leq c<1$ in the reactants.

When considering length scales $l \geq \delta_{L} \gg \eta_{K} \gg \delta_{r}$ (if $K a$ is sufficiently high in order for the latter inequality to hold), the thin reaction zone may be reduced to a reaction sheet. Then, the following constraints 57,99

$$
c\left(\boldsymbol{x}_{\boldsymbol{r}}, t\right)=1, \quad|\boldsymbol{n} \cdot \nabla c|_{r}=|\nabla c|_{r}=\left|\frac{\partial c}{\partial n}\right|_{r}=\frac{S_{L}}{D}=\frac{1}{\delta_{L}}
$$

hold at the reaction sheet. Accordingly, the reaction progress variable is continuous, but its gradient drops from $\delta_{L}^{-1}$ on the reactant side of the reaction sheet to zero on the product side. Equation (21) warrants that the reactant flux $D|\partial c / \partial n|_{r}$ towards the reaction sheet is equal to the rate $S_{L}$ of the reactant consumption per unit sheet area. Henceforth, subscript $r$ designates quantities or differential operators taken at the reactant side of the reaction sheet, $\boldsymbol{n}=-(\nabla c /|\nabla c|)_{r}$ is the unit vector normal to the reaction sheet, and $n$ is spatial distance counted from the reaction sheet along the $\boldsymbol{n}$-direction.

Under the above assumptions of $\rho=$ const, $v=$ const, single-step chemistry, and $L e=1$, the state of the mixture in the reaction wave is fully characterized by a single scalar variable ${ }^{99} c$ and the mixing zone $0<c(\boldsymbol{x}, t)<1$ in the reaction wave can be modeled by the standard diffusion equation

$$
\frac{\partial c}{\partial t}+\boldsymbol{u} \cdot \nabla c=D \Delta c
$$

Other initial and boundary conditions read $c\left(\boldsymbol{x}_{\boldsymbol{r}}, 0\right)=1, c(\boldsymbol{x}, t)=1$ in the products, and $c(x, y, z, t)=$ 0 when $x \rightarrow \infty$.

To close the problem and find a surface where the boundary condition given by Eq. (21) is stated, self-propagation of the reaction sheet $c\left(\boldsymbol{x}_{r}, t\right)=1$ and its advection by turbulent flow can be tracked using the displacement speed

$$
S_{d}^{r}=D(\Delta c /|\nabla c|)_{r}=D \delta_{L}(\Delta c)_{r}
$$

and the normal component $\boldsymbol{u}\left(\boldsymbol{x}_{r}, t\right) \cdot \boldsymbol{n}\left(\boldsymbol{x}_{r}, t\right)$ of the flow field $\boldsymbol{u}(\boldsymbol{x}, t)$. In an inhomogeneous flow, $S_{d}^{r}$ can significantly differ ${ }^{57}$ from $S_{L}$. For example, if term $D(\Delta c)_{r}$ is rewritten in the spherical coordinate system, the displacement speed $S_{d}^{r}$ involves an extra term whose magnitude $2 D / R_{r}$ is inversely proportional to the curvature radius $R_{r}$ of the reaction zone. Accordingly, if $R_{r}=\delta_{L}$ 
Publishiangd $(\partial c / \partial r)_{r}<0$ (the curvature center in products), the extra term overwhelms $S_{L}$ and makes $S_{d}^{r}$ negative. Strong variations in $S_{d}^{r}$ can also be caused by local velocity gradients even in a planar case $^{57}$. In the following theoretical analysis, the reaction sheet is not tracked and Eq. (23) is not used.

The problem stated above differs fundamentally from the classical problem of front propagation in a turbulent medium ${ }^{100,101}$. The point is that molecular mixing, i.e., the term on the Right Hand Side (RHS) of Eq. (22), is not directly addressed in the latter case. Accordingly, the latter problem is associated with $L \gg \delta_{L}$ and $D a \gg 1$, whereas the present paper addresses the case of a low $D a$. It is worth stressing that molecular mixing smooths out small-scale wrinkles of reaction-zone surface, generated by turbulent eddies, and, therefore, significantly reduces turbulent wave speed $S_{T}$. Indeed, if $S c=\mathrm{O}(1)$ and small-scale turbulent eddies are assumed to be able to wrinkle the reaction surface so that the local curvature radius $R_{r}$ of a wrinkle is on the order of the Kolmogorov length scale $\eta_{K}$, then the aforementioned mixing contribution $2 D / \eta_{K}$ to the displacement speed $S_{d}^{r}$ is locally comparable with the Kolmogorov velocity $v_{K}$ and is much larger than $S_{L}$ at $K a \gg 1$. Moreover, the mixing-controlled displacement speed $2 D / R_{r}$ locally affects the wrinkle even after dissipation of short-living Kolmogorov eddies that created the wrinkle. Consequently, the wrinkle is rapidly smoothed out. In other words, turbulence creates mixture non-uniformities of the Kolmogorov scale, followed by rapid dissipation of the non-uniformities by the molecular diffusion ${ }^{102}$. A recent DNS study ${ }^{47}$ does show that small-scale (when compared to $\delta_{L}$ ) wrinkles of a reaction-zone surface are efficiently smoothed out by molecular mixing, with this effect significantly reducing $S_{T}$ when compared to a linear dependence of $S_{T} \propto u^{\prime}$ simulated in the case of front propagation in the statistically same turbulence ${ }^{46}$.

\section{B. Can reaction affect the $c$-field when $D a$ is low?}

Within the framework of the problem stated above, the reaction term vanishes everywhere with exception of the reaction sheet and evolution of an iso-surface $c(\boldsymbol{x}, t)=\xi<1$ is described by the standard diffusion Eq. (22). Accordingly, the direct influence of the reaction on the evolution of the $c(\boldsymbol{x}, t)$-field is expected to be weak everywhere with exception of a narrow layer of $c^{*}<c \leq 1$ close to the reaction sheet.

To support this claim, let us compare magnitudes of (i) concentration gradient $|\nabla c|_{r}$ due to the reaction, i.e., $\delta_{L}^{-1}$, see Eq. (21), and (ii) concentration gradient $|\nabla c|_{T}$ due to inert turbulent mix- 
Publishijing. The latter magnitude may be estimated as follows $|\nabla c|_{T} \propto(\bar{N} / D)^{1 / 2}$. Since the mean scalar dissipation rate $\bar{N}=\overline{D(\nabla c)^{2}}$ is widely accepted ${ }^{2,102,103}$ to be independent of turbulent Reynolds number and to be proportional to $\tau_{T}^{-1}$ when $R e_{t} \gg 1$, we arrive at

$$
|\nabla c|_{T} \propto \frac{\left(S c R e_{t}\right)^{1 / 2}}{L} .
$$

Therefore, the ratio of the magnitudes of the two gradients scales as

$$
\frac{|\nabla c|_{r}}{|\nabla c|_{T}} \propto \frac{S_{L}}{D} L\left(S c R e_{t}\right)^{-1 / 2} \propto D a^{1 / 2} .
$$

Thus, if $D a \ll 1$ and $R e_{t} \gg 1$, scalar gradients generated by turbulent eddies are significantly larger than scalar gradients due to the reaction and, consequently, turbulent mixing overwhelms the influence of the reaction on the $c(x, t)$-field in the largest part of the mixing zone $0<c<1$. Nevertheless, the reaction appears to substantially affect the $c(\boldsymbol{x}, t)$-field in a narrow transition layer of $c^{*}<c \leq 1$ close to the reaction surface.

To estimate the thickness of the layer, let us expand $c(n)$ to Taylor series in the vicinity of the reaction sheet $c\left(\boldsymbol{x}_{r}, t\right)=1$

$$
c=1-\left|\frac{\partial c}{\partial n}\right|_{c=1} n+\left.\frac{1}{2} \frac{\partial^{2} c}{\partial n^{2}}\right|_{c=1} n^{2}+\mathrm{O}\left(n^{3}\right) .
$$

In the laminar reaction wave, the magnitude of the quadratic term is much less than the magnitude of the linear term if $n \ll \delta_{L}$. In a turbulent flow, small-scale eddies act to wrinkle the reaction sheet, thus, significantly increasing the magnitude of the quadratic term. Accordingly and since the expansion coefficient in the linear term is solely controlled by the reaction, see Eq. (21), let us assume that the thickness $n^{*}$ of the transition layer may be estimated by equating the linear and quadratic terms in Eq. (26), i.e.,

$$
n^{*} \approx 2\left(\delta_{L}\left|\frac{\partial^{2} c}{\partial n^{2}}\right|_{c=1}\right)^{-1}
$$

Then, to estimate the order of magnitude of the second derivative $\left|\partial^{2} c / \partial n^{2}\right|_{c=1}$ in a turbulent flow, let us consider the simplest relevant model problem ${ }^{57,104}$, i.e., an 1D planar laminar reaction wave stabilized in a 2D flow $\{u=-\gamma x, v=\gamma y\}$, with the velocity gradient $\gamma$ being on the order of $\tau_{K}^{-1}$. In such a case, Eq. (22) reads

$$
-\frac{x}{\tau_{K}} \frac{\mathrm{d} c}{\mathrm{~d} x}=D \frac{\mathrm{d}^{2} c}{\mathrm{~d} x^{2}}
$$


Publishifigs. (21) holds, and other boundary conditions are $c\left(x_{0}\right)=1$ and $c(-\infty)=0$, where $x_{0}$ is the coordinate of the reaction zone and $n=x_{0}-x$. If $\tau_{K} \ll \delta_{L}^{2} / D$, i.e., $K a \gg 1$, integration of Eq. (28) results in 104

$$
x_{0} \approx S c^{-1 / 2} \eta_{K} \sqrt{\ln (K a / 2 \pi)}
$$

Moreover, at the reaction surface $x=x_{0}$, Eqs. (21) and (28) yield

$$
\left.\frac{\partial^{2} c}{\partial n^{2}}\right|_{c=1}=-\frac{S c}{\eta_{K}^{2}} \frac{x_{0}}{\delta_{L}} .
$$

Finally, combining Eqs. (27), (29), and (30), we arrive at

$$
n^{*} \approx \frac{2 \eta_{K}}{\sqrt{S c \ln (K a / 2 \pi)}} \ll S c^{-1 / 2} \eta_{K} .
$$

At the boundary of the transition layer, the difference between unity and the boundary value $c^{*}$ of the reaction progress variable is less than $\varepsilon=2 / \sqrt{K a \ln (K a / 2 \pi)} \ll 1$, see the second, linear, term on the RHS of Eq. (26) and note that the positive third, quadratic, term makes the difference even smaller. In order for the distance $n_{r}$ to be much larger than the reaction-zone thickness, Ze should be much lager than $\sqrt{S c K a \ln (K a / 2 \pi)}$ provided that $\delta_{r}=\mathrm{O}\left(\delta_{L} / Z e\right)$.

\section{Turbulent consumption velocity}

The above order-of-magnitude estimates support the following scenario. If $D a \ll 1, K a \gg 1$, $R e_{t} \gg 1$, and $S c=\mathrm{O}(1)$, an infinitely fast reaction can significantly affect the $c(\boldsymbol{x}, t)$-field solely in a narrow layer in the vicinity of the infinitely thin reaction zone, with the thickness of this layer being less than the Kolmogorov scale $\eta_{K}$. Since distance $n^{*}$ between the reaction sheet $c\left(\boldsymbol{x}_{r}, t\right)=1$ and the iso-surface $c(\boldsymbol{x}, t)=c^{*}<1$ that bounds the transition layer is so small, we may assume that the two surfaces move in a close correlation with one another and, hence, their areas $A_{r}$ and $A_{c^{*}}$ are approximately equal to the leading order. Furthermore, since the evolution of the latter isosurface is argued to be weakly affected by the reaction, the latter area can be estimated as follows $A_{c^{*}} \propto A_{c}$ invoking knowledge on the area $A_{c}$ of an iso-scalar surface in the case of inert turbulent mixing. This is the key point of the present approach.

Thus, turbulent consumption velocity, i.e., bulk rate $Q$ of reactant consumption normalized with the density and the area $A_{0}$ of the mean reaction-wave surface, scales as

$$
U_{T}=\frac{Q}{\rho A_{0}}=S_{L} \frac{A_{c^{*}}}{A_{0}} \propto S_{L} \frac{A_{c}}{A_{0}} .
$$


PublishiMg eover, by hypothesizing independence of the PDF $P(c)$ and mean scalar dissipation rate $\bar{N}$ on turbulent Reynolds number at $R e_{t} \gg 1$, Kuznetsov and Sabelnikov ${ }^{2}$ have obtained the following scaling

$$
A_{c} \propto A_{0}\left(S c R e_{t}\right)^{1 / 2}
$$

for the area of an inert iso-scalar surface. The same scaling results from widely accepted independence ${ }^{105,106}$ of the bulk inert scalar flux $F_{c}$ through an iso-surface $c(\boldsymbol{x}, t)=$ const on turbulent Reynolds number at $R e_{t} \gg 1$. Indeed, since

$$
F_{c}=D \int_{A_{c}}\left|\frac{\partial c}{\partial n}\right|_{T} \mathrm{~d} A_{c} \propto \frac{D}{L}\left(S c R e_{t}\right)^{1 / 2} A_{c} \propto u^{\prime} A_{c}\left(S c R e_{t}\right)^{-1 / 2}
$$

see Eq. (24), $A_{c}$ should be proportional to $\left(S c R e_{t}\right)^{1 / 2}$ in order for the flux $F_{c}$ to be independent of $R e_{t}$. It is worth noting that the flux $F_{c}$ is controlled by the relative velocity of the surface $c(x, t)=$ const with respect to the local flow, with the relative velocity being solely controlled by the molecular diffusion. Accordingly, in the case of a material surface, $D=F_{c}=0$ and neither Eq. (33) nor Eq. (34) holds.

Finally, Eq. (32) and $A_{c} / A_{0} \propto\left(S c R e_{t}\right)^{1 / 2}$ yield the following scaling

$$
U_{T} \propto S_{L}\left(S c R e_{t}\right)^{1 / 2}=u^{\prime} D a^{1 / 2}
$$

for the turbulent consumption velocity $U_{T}$. This scaling is basically similar to Damköhler's classical scaling given by Eq. (4).

\section{Mean wave brush thickness}

In order to arrive at Eq. (35), we hypothesized (based on physical reasoning and estimates) that the field of $c(\boldsymbol{x}, t)$ is substantially affected by the reaction solely in a narrow (if $D a \ll 1$ and $\left.R e_{t} \gg 1\right)$ layer close to the reaction sheet. This assumption might be put into question by pointing out that, since evolution of an iso-surface $c(x, t)=\xi<c^{*}$ is not affected by the reaction, mean turbulent wave-brush thickness $\delta_{T}$ should permanently grow, similarly to the mean thickness of a turbulent mixing layer.

However, it is worth remembering the following important difference between inert mixing and propagation of a reaction sheet. In the former case, the mean position, e.g., $\bar{c}(x, t)=0.5$, of the mixing layer is constant in the coordinate framework attached to the mean flow, whereas the mean 
Publishiregction-wave surface propagates in such a framework. Accordingly, we may assume that the mean wave-brush thickness reaches the fully developed state when the speed of the leading edge of the inert mixing layer is equal to the mean speed $S_{T}$ of the wave, which is equal to $U_{T}$ in the considered statistically planar 1D case. Since the growth of the mean thickness of a turbulent mixing layer follows the turbulent diffusion law, i.e., $\delta_{T} \propto\left(u^{\prime} L t\right)^{1 / 2}$ if $t \gg \tau_{T}^{107,108}$, time $t_{\delta}$ required for the mean wave brush to reach the fully developed state may be estimated as

$$
\left(\frac{u^{\prime} L}{t_{\delta}}\right)^{1 / 2} \approx S_{L}\left(\operatorname{SCRe}_{t}\right)^{1 / 2}
$$

using Eq. (35). Consequently,

$$
t_{\delta} \approx \tau_{L}=\frac{\tau_{T}}{D a} \gg \tau_{T}
$$

and

$$
\delta_{T} \propto\left(u^{\prime} L t_{\delta}\right)^{1 / 2} \propto \delta_{L}\left(S c R e_{t}\right)^{1 / 2}=L D a^{-1 / 2} .
$$

This scaling is qualitatively consistent with the present DNS data in the sense that $\delta_{T} / L$ increases with decreasing $D a$. However, the theoretical scaling (power) exponent differs from the scaling exponent that fits the entire set of the present DNS data, see the black dotted-dashed line in Fig. 9. Nevertheless, if solely DNS data characterized by the lowest $D a \leq 0.023$ are considered, see the red solid line in the same figure, then, the theoretical and numerical scaling exponents agree well.

It is of interest to note that the present analysis, see Eqs. (33) and (38), yields the following scaling

$$
\frac{1}{\delta_{T}} \int_{-\infty}^{\infty} \bar{\Sigma} \mathrm{d} x=\frac{A_{r}}{\delta_{T} A_{0}} \propto \frac{A_{c^{*}}}{\delta_{T} A_{0}} \propto \frac{A_{c}}{\delta_{T} A_{0}} \propto \frac{1}{\delta_{L}}
$$

for the integrated reaction surface density $\bar{\Sigma}$. While this result might appear to be unexpected due to the lack of turbulence characteristics on the RHS of Eq. (39), it is consistent with the above reasoning that molecular mixing efficiently smooths out small-scale wrinkles of the reaction sheet. Indeed, Eq. (39) implies that the mean distance between reaction surfaces is on the order of the laminar wave thickness, i.e., the distance is directly controlled by molecular diffusivity. Since the aforementioned reasoning were early used to stress importance of the mixing and to argue significant differences in $S_{L}$ and $S_{d}^{r}$, but were not used to obtain Eqs. (35) and (38), the scaling given by Eq. (39) indicates self-consistency of the present analysis.

It is also of interest to note that, similarly to Eq. (35) which coincides with Damköhler's scaling for $U_{T}$, Eq. (38) coincides with Damköhler's scaling for $\delta_{T}$ in spite of the fact that physical mechanisms highlighted by Damköhler ${ }^{9}$ and in the present work are substantially different. 
Thin reaction Tqghanuscript was accepted by Phys. Fluids. Click here to see the version of record.

Equation (35) is consistent with the present DNS data computed for low $D a$ and high $K a$, see Fig. 3(a), and explains the weak influence of $Z e$ on $U_{T}$ in the present and other ${ }^{60}$ simulations. Indeed, within the framework of the developed theory, an increase in the reaction-sheet area is controlled by turbulent mixing of the reactants and products. Application of the same hypothesis to the case of a thin reaction zone of a finite thickness implies that the reaction-zone-surface area and, hence, $U_{T} / S_{L}$ are weakly affected by the structure of the reaction zone and, in particular, by Ze at a low $D a$.

Moreover, Eq. (35) is consistent with experimental data obtained for reaction-front propagation in aqueous solutions ${ }^{1}$ and premixed turbulent flames ${ }^{32}$, as well as with DNS obtained from constant-density single-reaction waves in $2 \mathrm{D}$ turbulence ${ }^{33}, 3 \mathrm{D}$ thermonuclear deflagration waves ${ }^{8}$, and 3D highly turbulent lean methane-air and hydrogen-air flames ${ }^{34}$. Furthermore, the present theoretical analysis is also consistent with experimental $31,36,42,68,69$ and $\mathrm{DNS}^{34,70-79}$ observations of thin heat-release zones in flames characterized by low $\mathrm{Da}$ and high $\mathrm{Ka}$.

The present theoretical analysis does not seem to be contradicted by DNS data ${ }^{7,8,75,80}$ that (i) indicate that the influence of the Lewis number on the local instantaneous structure of flames in highly turbulent flows reduces with increasing $K a$ and, therefore, (ii) imply a more important role played by turbulent transport at high $K a$, in line with the hypothesis on mixing enhancement by turbulence. However, such data prove neither statistically important broadening of reaction zones nor turbulence-controlled mixing within the zones. Indeed, according to the theory of stretched laminar premixed flames ${ }^{99,109}$, the influence of the Lewis number on the local consumption velocity and reaction zone structure is mainly controlled by molecular fluxes towards/from the zone, rather than by/mixing within the zone. Accordingly, if (i) the molecular heat diffusivity $\kappa$ and molecular diffusivities of main reactants are different, (ii) turbulent mixing dominates in preheat zones of stretched flames, thus, reducing importance of these differences, but, (iii) within the flame reaction zones, the local mixing is controlled by molecular transport; then, the structure of the reaction zones is likely to be changed anyway. Therefore, the aforementioned DNS data ${ }^{7,8,75,80}$ do not seem to be a decisive proof of turbulence-controlled mixing within reaction zones.

While Eq. (35) coincides with Damköhler's classical scaling, the two results (i) were obtained invoking different assumptions, i.e., $L \ll \delta_{L}$ in Damköhler's paper ${ }^{9}$, but $D a \ll 1$ in the present work, and (ii) are associated with different regimes, i.e., DRZs and TRZs, respectively. The gov- 
Publishiørging physical mechanisms are different within the framework of the DRZ concept and in the present work, i.e., mixing enhancement by turbulent eddies within broad reaction zones and an increase in the area of reaction-zone surface due to turbulent mixing outside the reaction zone, respectively. A common feature of the two approaches consists in highlighting an important role played by turbulent mixing.

In spite of the noted differences between the DRZ concept and the present study, the study does not contradict to Damköhler's classical work. The point is that Damköhler himself did hypothesize mixing enhancement by turbulent eddies within a reaction wave, did reduce modeling of such effects solely to substitution of molecular diffusivity $D$ with turbulent diffusivity $D_{T}$, but did not discuss reaction-zone broadening. Accordingly, his general concept is consistent both with the present theory and with the present DNS data, but the concept does not reveal the governing physical mechanism of the influence of intense turbulence on burning rate. The concept admits both (i) an increase in the reaction-zone surface, controlled by turbulent mixing ahead of the surface, which is consistent with the present theory and DNS data, and (ii) mixing enhancement within reaction zones, which is ignored by the present theory and is not highlighted by the present DNS data.

Finally, it is worth noting that the present theory has certain common features with Peters' concept of TRZs. In particular, reaction zones are thin and their surface area is controlled by mixing in both cases. Nevertheless, there are substantial differences also. For instance, Eqs. (19) and (35) were obtained using different methods and, in particular, ill-substantiated Eq. (20) was not invoked for this purpose in the present study.

\section{CONCLUSIONS}

The major results of the present work are as follows.

First, Damköhler's classical scaling given by Eq. (4), which is commonly associated with distributed reaction zones, is numerically and theoretically shown to be also consistent with propagation of thin reaction zones in intense turbulence at $D a<1$ and $K a \gg 1$.

Second, under conditions simulated in the paper ( $D a$ is as low as 0.01 and $K a$ is as large as about 600), consumption rate is mainly controlled by the area of the surface of a thin reaction zone.

Third, the present numerical results are consistent with the concept of thin-reaction-zone 
Publishinegime, introduced by Peters ${ }^{3}$, but show that the regime expands to values of $K a$ that are much higher than a critical value $K a^{*}$ estimated by simply comparing the reaction-zone thickness and the Kolmogorov length scale.

Fourth, a new simple phenomenological theory of the TRZ regime is developed and validated using the present DNS data, as well as recent experimental and DNS results.

All in all, the main message provided by this work is that reactions may be confined to thin zones even at a very high $K a$ and a low $D a$, with Eq. (4) holding under such conditions. Accordingly, the present study offers an opportunity to reconcile (i) recent experimental ${ }^{31,36,42,68,69}$ and DNS ${ }^{34,70-79}$ data that indicate statistically weak (or the lack of) reaction-zone broadening in flames characterized by high $K a$ and low $D a$, and (ii) recent experimental ${ }^{32}$ and $\mathrm{DNS}^{8,33,34}$ data that support Eq. (4) at high $K a$ and low $D a$.

Moreover, the present DNS data indicate that the profile $\bar{W}(\bar{c})$ of the mean reaction rate vs. the mean reaction progress variable approaches the laminar profile $W(\bar{c})$ with decreasing $D a$. This trend offers an opportunity to significantly simplify modeling of the influence of intense turbulence on reactions.

The DNS data also show that the mean wave brush thickness normalized using a turbulence length scale, e.g. $\delta_{T} / L_{11}$, increases with decreasing $D a$. If (i) the data are fitted by $\delta_{T} / L_{11} \propto D a^{q}$ in a range $D a<D a^{*}$ of low Damköhler numbers and (ii) the range boundary $D a^{*}$ is decreased, then the power exponent $q$ approaches $q=-0.5$, which results from both Damköhler's classical hypothesis and the present theory.

Furthermore, the DNS data evidence that, in line with the present theory, the thickness of the reaction zone in a laminar flow weakly affects $U_{T} / u^{\prime}$, provided that $D a$ is kept constant and the thickness is small.

Finally, the present DNS data indicate that contributions of inclination and folding to the reaction-surface-area increase are comparable for moderately large ratios $S c R e_{t}$ of turbulent and molecular diffusivities. When $S c R e_{t}$ is increased, the magnitude of the folding contribution is also increased but the magnitude of the inclination contribution levels-off. Accordingly, the folding contribution is of the most importance at the largest $S c R e_{t}$ reached in the simulations. The magnitude of the broadening contribution is substantially lower than the magnitude of the inclination contribution in the entire range of addressed $S c R e_{t}$.

When applying the above results to more complicated problems, e.g., to premixed turbulent combustion, it is worth remembering important effects, e.g., thermal expansion, preferential dif- 
Publishifugion, complex chemistry, or/and heat losses, that vanish within the framework of the simple problem addressed in the present paper.

\section{ACKNOWLEDGMENTS}

VAS gratefully acknowledges the financial support by ONERA and by the Grant of the Ministry of Education and Science of the Russian Federation (Contract No. 14.G39.31.0001 of 13.02.2017). RY gratefully acknowledges the financial support by the Swedish Research Council. ANL gratefully acknowledges the financial support by the Combustion Engine-Research Center (CERC). The simulations were performed using the computer facilities provided by the Swedish National Infrastructure for computing (SNIC) at Beskow-PDC center.

\section{REFERENCES}

${ }^{1}$ P. D. Ronney, B. D., Haslam, and N. O. Rhys, "Front propagation rates in randomly stirred media," Phys. Rev. Lett. 74, 3804 (1995).

${ }^{2}$ V. R. Kuznetsov and V. A. Sabelnikov, Turbulence and Combustion (Hemisphere Publ. Corp., New York, 1990).

${ }^{3}$ N. Peters, Turbulent Combustion (Cambridge University Press, Cambridge, UK, 2000).

${ }^{4}$ A. Lipatnikov, Fundamentals of Premixed Turbulent Combustion (CRC Press, Boca Raton, Florida, 2012).

${ }^{5}$ A. Y. Poludnenko, T. A. Gardiner, and E. S. Oran, "Spontaneous transition of turbulent flames to detonations in unconfined media," Phys. Rev. Lett. 107, 054501 (2011).

${ }^{6}$ V. N. Gamezo, A. M. Khokhlov, E. S. Oran, A. Y. Chtchelkanova, and R. O. Rosenberg, "Thermonuclear supernovae: Simulations of the deflagration stage and their implications," Science 299, 77 (2003).

${ }^{7}$ A. J. Aspden, J. B. Bell, M. S. Day, S. E. Woosley, and M. Zingale, "Turbulence-flame interactions in type Ia supernovae," Astrophys. J. 689, 1173 (2008).

${ }^{8}$ A. J. Aspden, J. B. Bell, and S. E. Woosley "Distributed flames in type Ia supernovae," Astrophys. J. 710, 1654 (2010).

${ }^{9}$ G. Damköhler, "Der einfuss der turbulenz auf die flammengeschwindigkeit in gasgemischen," Z. Electrochem. 46, 601 (1940). 
PublishingR. Borghi, "On the structure and morphology of turbulent premixed flames," in Recent Advances in Aerospace Science, edited by S. Casci and C. Bruno (Plenum, New York, 1984) p. 117.

${ }^{11}$ F. A. Williams, Combustion Theory (Benjamin/Cummings, Menlo Park, California, 1985) 2nd ed.

${ }^{12}$ N. Peters, "Laminar flamelet concepts in turbulent combustion," Proc. Combust. Inst. 21, 1231 (1986).

${ }^{13}$ A. N. Lipatnikov and J. Chomiak, "Molecular transport effects on turbulent flame propagation and structure," Prog. Energy Combust. Sci. 31, 1 (2005).

${ }^{14}$ P. Venkateswaran, A. Marshall, J. Seitzman, and T. Lieuwen, "Scaling turbulent flame speeds of negative Markstein length fuel blends using leading points concepts," Combust. Flame 162, 375 (2015).

${ }^{15}$ S. Yang, A. Saha, W. Liang, F. Wu, and C. K. Law, "Extreme role of preferential diffusion in turbulent flame propagation," Combust. Flame 188, 498 (2018).

${ }^{16}$ K. N. C. Bray, "Turbulent transport in flames," Proc. R. Soc. London A 451, 231 (1995).

${ }^{17}$ A. N. Lipatnikov and J. Chomiak, "Effects of premixed flames on turbulence and turbulent scalar transport," Prog. Energy Combust. Sci. 36, 1 (2010).

${ }^{18}$ N. Fogla, F. Creta, and M. Matalon, "The turbulent flame speed for low-to-moderate turbulence intensities: Hydrodynamic theory vs. experiments," Combust. Flame 175, 155 (2017).

${ }^{19}$ V. A. Sabelnikov and A. N. Lipatnikov, "Recent advances in understanding of thermal expansion effects in premixed turbulent flames," Annu. Rev. Fluid Mech. 49, 91 (2017).

${ }^{20} \mathrm{H}$. Carlsson, R. Yu, and X. S. Bai, "Direct numerical simulation of lean premixed $\mathrm{CH}_{4} / \mathrm{air}$ and $\mathrm{H}_{2}$ /air flames at high Karlovitz numbers," Int. J. Hydrogen Energy 39, 20216 (2014).

${ }^{21}$ A. J. Aspden, M. S. Day, and J. B. Bell, "Turbulence-chemistry interaction in lean premixed hydrogen combustion,” Proc. Combust. Inst. 35, 1321 (2015).

${ }^{22}$ D. Dasgupta, W. Sun, M. Day, and T. C. Lieuwen, "Effect of turbulence-chemistry interactions on chemical pathways for turbulent hydrogen-air premixed flames," Combust. Flame 176, 191 (2017).

${ }^{23}$ T. Poinsot, D. Veynante, and S. Candel, "Quenching processes and premixed turbulent combustion diagrams," J. Fluid Mech. 228, 561 (1991).

${ }^{24}$ A. N. Lipatnikov and J. Chomiak, "Turbulent flame speed and thickness: Phenomenology, evaluation, and application in multi-dimensional simulations," Prog. Energy Combust. Sci. 28, 
Thin reaction Tqnafanuscript was accepted by Phys. Fluids. Click here to see the version of record.

\section{Publishing1 (2002).}

${ }^{25}$ D. Veynante and L. Vervisch, “Turbulent combustion modeling,” Prog. Energy Combust. Sci. 28, 193 (2002).

${ }^{26}$ S. Chaudhuri, V. Akkerman, and C. K. Law, "Spectral formulation of turbulent flame speed with consideration of hydrodynamic instability," Phys. Rev. E 84, 026322 (2011).

${ }^{27}$ E. S. Shetinkov, “Calculation of flame velocity in turbulent stream," Proc. Combust. Inst. 7, 583 (1958).

${ }^{28}$ A. S. Monin and A. M. Yaglom, Statistical Fluid Mechanics: Mechanics of Turbulence (The MIT Press, Cambridge, Massachusetts, 1975) Vol. 2.

${ }^{29}$ L. D. Landau and E. M. Lifshitz, Fluid Mechanics (Pergamon Press, New York, 1987).

${ }^{30}$ K. I. Shchelkin, “On combustion in a turbulent flow," NACA TM, 1110 (1947).

${ }^{31}$ A. W. Skiba, T. M. Wabel, C. D. Carter, S. D. Hammack, J. E. Temme, and J. F. Driscoll, "Premixed flames subjected to extreme levels of turbulence part I: Flame structure and a new measured regime diagram," Combust. Flame 189, 407 (2018).

${ }^{32}$ S. S. Shy, C. C. Liu, J. Y. Lin, L. L. Chen, A. N. Lipatnikov, and S. I. Yang, "Correlations of high-pressure lean methane and syngas turbulent burning velocities: Effects of turbulent Reynolds, Damköhler, and Karlovitz numbers," Proc. Combust. Inst. 35, 1509 (2015).

${ }^{33}$ C. R. Koudella and Z. Neufeld, "Reaction front propagation in a turbulent flow," Phys. Rev. E 70, 026307 (2004).

${ }^{34}$ A. J. Aspden, M. S. Day, and J. B. Bell, "Towards the distributed burning regime in turbulent premixed flames,' e-print arXiv:1806.09865.v1 (2018).

${ }^{35}$ T. M. Wabel, A. W. Skiba, and J. F. Driscoll, “Turbulent burning velocity measurements: Extended to extreme levels of turbulence," Proc. Combust. Inst. 36, 1801 (2017).

${ }^{36}$ T. M. Wabel, A. W. Skiba, J. E. Temme, and J. F. Driscoll, "Measurements to determine the regimes of premixed flames in extreme turbulence," Proc. Combust. Inst. 36, 1809 (2017).

${ }^{37}$ A. W. Skiba, T. M. Wabel, C. D. Carter, S. D. Hammack, J. E. Temme, T. Lee, and J. F. Driscoll, "Reaction layer visualization: A comparison of two PLIF techniques and advantages of kHzimaging," Proc. Combust. Inst. 36, 4593 (2017).

${ }^{8}$ T. M. Wabel, A. W. Skiba, and J. F. Driscoll, "Evolution of turbulence through a broadened preheat zone in a premixed piloted Bunsen flame from conditionally-averaged velocity measurements," Combust. Flame 188, 13 (2018).

${ }^{39}$ Z. S. Li, B. Li, Z. W. Sun, X.-S. Bai, and M. Aldén, “Turbulence and combustion interaction: 
Publishing High resolution local flame front structure visualization using simultaneous single-shot PLIF imaging of $\mathrm{CH}, \mathrm{OH}$, and $\mathrm{CH}_{2} \mathrm{O}$ in a piloted premixed jet flame," Combust. Flame 157, 1087 (2010).

${ }^{40}$ B. Zhou, C. Brackmann, Z. Li, M. Aldén, and X.-S. Bai, "Simultaneous multi-species and temperature visualization of premixed flames in the distributed reaction zone regime," Proc. Combust. Inst. 35, 1409 (2015).

${ }^{41}$ B. Zhou, C. Brackmann, Q. Li, Z. Wang, P. Petersson, Z. Li, M. Aldén, and X.-S. Bai, “Distributed reactions in highly turbulent premixed methane/air flames. Part I. Flame structure characterization," Combust. Flame 162, 2937 (2015).

${ }^{42}$ B. Zhou, C. Brackmann, Z. Wang, Z. Li, M. Richter, M. Aldén, and X.-S. Bai, “Thin reaction zone and distributed reaction zone regimes in turbulent premixed methane/air flames: Scalar distributions and correlations," Combust. Flame 175, 220 (2017).

${ }^{43}$ M. J. Dunn, A. R. Masri, R. W. Bilger, and R. S. Barlow, "Finite rate chemistry effects in highly sheared turbulent premixed flames," Flow Turbul. Combust. 85, 621 (2010).

${ }^{44}$ B. Coriton and J. H. Frank, "Experimental study of vorticity-strain rate interaction in turbulent partially premixed jet flames using tomographic particle image velocimetry,” Phys. Fluids 28, 025109 (2016).

${ }^{45}$ R. Yu, A. N. Lipatnikov, and X. S. Bai, "Three-dimensional direct numerical simulation study of conditioned moments associated with front propagation in turbulent flows," Phys. Fluids 26, 085104 (2014).

${ }^{46}$ R. Yu, X. S. Bai, and A. N. Lipatnikov, "A direct numerical simulation study of interface propagation in homogeneous turbulence,” J. Fluid Mech. 772, 127 (2015).

${ }^{47}$ R. Yu and A. N. Lipatnikov, "Direct numerical simulation study of statistically stationary propagation of areaction wave in homogeneous turbulence," Phys. Rev. E 95, 063101 (2017).

${ }^{48}$ R. Yu and A. N. Lipatnikov, "DNS study of dependence of bulk consumption velocity in a constant-density reacting flow on turbulence and mixture characteristics," Phys. Fluids 29, 065116 (2017).

${ }^{49}$ T. Elperin, N. Kleeorin, M. Liberman, A. N. Lipatnikov, I. Rogachevskii, and R. Yu, “Turbulent diffusion of chemically reacting flows: Theory and numerical simulations," Phys. Rev. E 96, 053111 (2017).

${ }^{50}$ V. A. Sabelnikov, R. Yu, and A. N. Lipatnikov, "Thin reaction zones in highly turbulent medium,” Int. J. Heat Mass Transfer 128, 1201 (2019). 
Thin reaction Tonganuscript was accepted by Phys. Fluids. Click here to see the version of record.

PublishingfR. Yu and A. N. Lipatnikov, "A DNS study of sensitivity of scaling exponents for premixed turbulent consumption velocity to transient effects," Flow Turbulence Combust., in press.

${ }^{52}$ R. Yu, J. Yu, and X. S. Bai, “An improved high-order scheme for DNS of low Mach number turbulent reacting flows based on stiff chemistry solver," J. Comp. Phys. 231, 5504 (2012).

${ }^{53}$ G. S. Jiang and C. W. Shu, "Efficient implementation of weighted ENO schemes,” J. Comput. Phys. 126, 202 (1996).

${ }^{54}$ R. Yu and X. S. Bai, "A fully divergence-free method for generation of inhomogeneous and anisotropic turbulence with large spatial variation,” J. Comp. Phys. 256, 234 (2014).

${ }^{55}$ V. Eswaran and S. B. Pope, "An examination of forcing in direct numerical simulations of turbulence," Comput. Fluids 16, 257 (1988).

${ }^{56}$ A. G. Lamorgese, D. A. Caughey, and S. B. Pope, "Direct numerical simulation of homogeneous turbulence with hyperviscosity," Phys. Fluids 17, 015106 (2005).

${ }^{57}$ A. M. Klimov, "Laminar flame in a turbulent flow," Zh. Prikl. Mekch. Tekhn. Fiz. 4, No. 3, 49 (1963).

${ }^{58}$ K. N. C. Bray and R. S. Cant, "Some applications of Kolmogorov's turbulence research in the field of combustion,” Proc. R. Soc. London A 434, 217 (1991).

${ }^{59}$ D. Bradley, "How fast can we burn?" Proc. Combust. Inst. 24, 247 (1992).

${ }^{60}$ A. N. Lipatnikov, V. A. Sabelnikov, and A. Y. Poludnenko, "Assessment of a transport equation for mean reaction rate using DNS data obtained from highly unsteady premixed turbulent flames,” Int. J. Heat/Mass Transfer 134, 398 (2019).

${ }^{61}$ C. Duwig, K. J. Nogenmyr, C. K. Chan, and M. J. Dunn, "Large eddy simulations of a piloted lean premix jet flame using finite-rate chemistry," Combust. Theory Modelling 15, 537 (2011).

${ }^{62}$ G. V. Nivarti and R. S. Cant, "Direct numerical simulation of the bending effect in turbulent premixed flames," Proc. Combust. Inst. 36, 1903 (2017).

${ }^{63}$ S. Lapointe and G. Blanquart, "Fuel and chemistry effects in high Karlovitz premixed turbulent flames," Combust. Flame 167, 394 (2016).

${ }^{64}$ Z. Wang, V. Magi, and J. Abraham, "Turbulent flame speed dependencies of lean methane-air mixtures under engine relevant conditions," Combust. Flame 180, 53 (2017).

${ }^{65}$ A. A. Burluka, J. F. Griffiths, K. Liu, and M. Orms, "Experimental studies of the role of chemical kinetics in turbulent flames," Combust. Explos. Shock Waves 45, 383 (2009).

${ }^{66}$ A. N. Lipatnikov, W. Y. Li, L. J. Jiang, and S. S. Shy, "Does density ratio significantly affect turbulent flame speed?" Flow Turbul. Combust. 98, 1153 (2017). 
Publishinģ A. N. Lipatnikov, J. Chomiak, V. A. Sabelnikov, S. Nishiki, and T. Hasegawa, "A DNS study of the physical mechanisms associated with density ratio influence on turbulent burning velocity in premixed flames," Combust. Theory Modelling 22, 131 (2018).

${ }^{68}$ J. F. Driscoll, “Turbulent premixed combustion: Flamelet structure and its effect on turbulent burning velocities,” Prog. Energy Combust. Sci. 34, 91 (2008).

${ }^{69}$ B. R. Chowdhury and B. M. Cetegen, "Experimental study of the effects of free stream turbulence on characteristics and flame structure of bluff-body stabilized conical lean premixed flames," Combust. Flame 178, 311 (2017).

${ }^{70}$ A. Y. Poludnenko and E. S. Oran, “The interaction of high-speed turbulence with flames: Global properties and internal flame structure," Combust. Flame 157, 995 (2010).

${ }^{71}$ P. E. Hamlington, A. Y. Poludnenko, and E. S. Oran, "Interactions between turbulence and flames in premixed reacting flows," Phys. Fluids 23, 125111 (2011).

${ }^{72}$ P. E. Hamlington, A. Y. Poludnenko, and E. S. Oran, "Intermittency in premixed turbulent reacting flows," Phys. Fluids 24, 075111 (2012).

${ }^{73}$ D. Thévenin, "Three-dimensional direct simulations and structure of expanding turbulent methane flames," Proc. Combust. Inst. 30, 629 (2005).

${ }^{74}$ R. Sankaran, E. R. Hawkes, C. S. Yoo, and J. H. Chen, "Response of flame thickness and propagation speed under intense turbulence in spatially developing lean premixed methane-air jet flames," Combust. Flame 162, 3294 (2015).

${ }^{75}$ S. Lapointe, B. Sayard, and G. Blanquart, "Differential diffusion effects, distributed burning, and local extinction in high/Karlovitz premixed flames," Combust. Flame 162, 3341 (2015).

${ }^{76}$ A. J. Aspden, M. S. Day, and J. B. Bell, "Three-dimensional direct numerical simulation of turbulent lean premixed methane combustion with detailed kinetics," Combust. Flame 165, 266 (2016).

${ }^{77}$ S. Chaudhuri, H. Kolla, H. L. Dave, E. R. Hawkes, J. H. Chen, and C. K. Law, "Flame thickness and conditional scalar dissipation rate in a premixed temporal turbulent reacting jet," Combust. Flame 184, 273 (2017).

${ }^{78}$ T. Nilsson, H. Carlsson, R. Yu, and X.-S. Bai, "Structures of turbulent premixed flames in the high Karlovitz number regime - DNS analysis," Fuel 216, 627 (2017).

${ }^{79}$ H. Wang, E. R. Hawkes, J. H. Chen, B. Zhou, Z. Li, and M. Aldén, "Direct numerical simulations of a high Karlovitz number laboratory premixed jet flame - an analysis of flame stretch and flame thickening," J. Fluid Mech. 815, 511 (2017). 
Thin reaction Tonganuscript was accepted by Phys. Fluids. Click here to see the version of record.

PublishingeA. J. Aspden, M. S. Day, and J. B. Bell, “Turbulence-flame interactions in lean premixed hydrogen: Transition to the distributed burning regime," J. Fluid Mech. 680, 287 (2011).

${ }^{81}$ W. L. Roberts, J. F. Driscoll, M. C. Drake, and L. P. Goss, "Images of the quenching of a flame by vortex - to quantify regimes of turbulent combustion," Combust. Flame 94, 58 (1993).

${ }^{82}$ P. H. Renard, D. Thévenin, J. C. Rolon, and S. Candel, "Dynamics of flame/vortex interactions," Prog. Energy Combust. Sci. 26, 225 (2000).

${ }^{83}$ S. Kadowaki and T. Hasegawa, "Numerical simulation of dynamics of premixed flames: Flame instability and vortex-flame interaction,” Prog. Energy Combust. Sci. 31, 193 (2005).

${ }^{84}$ A. N. Lipatnikov, S. Nishiki, and T. Hasegawa, "A direct numerical simulation study of vorticity transformation in weakly turbulent premixed flames," Phys. Fluids 26, 105104 (2014).

${ }^{85}$ B. Bobbitt, S. Lapointe, and G. Blanquart, "Vorticity transformation in high Karlovitz number premixed flames," Phys. Fluids 28, 015101 (2014).

${ }^{86}$ S. H. R. Whitman, C. A. Z. Towery, A. Y. Poludnenko, and P. E. Hamlington, "Scaling and collapse of conditional velocity structure functions in turbulent premixed flames," Proc. Combust. Inst. 37, 2527 (2019).

${ }^{87}$ V. P. Karpov and A. S. Sokolik, "Ignition limits in turbulent gas mixtures," Proc. Acad. Sci. USSR, Phys. Chem. 141, 866 (1961).

${ }^{88}$ V. P. Karpov and E. S. Severin, "Effects of molecular-transport coefficients on the rate of turbulent combustion,” Combust. Explos. Shock Waves 16, 41 (1980).

${ }^{89}$ R. G. Abdel-Gayed, K. J. Al-Khishali, and D. Bradley, "Turbulent burning velocities and flame straining in explosions," Proc. R. Soc. London A 391, 391 (1984).

${ }^{90}$ S. I. Yang and S. S. Shy, "Global quenching of premixed $\mathrm{CH}_{4} /$ air flames: Effects of turbulent straining, equivalence ratio, and radiative heat loss," Proc. Combust. Inst. 29, 1841 (2002).

${ }^{91}$ J. Kariuki, A. Dowling, R. Yuan, R. Balachandran, and E. Mastorakos, "Heat release imaging in turbulent premixed methane/air flames close to blow-off," Proc. Combust. Inst. 35, 1443 (2015).

${ }^{92}$ B. Zhou, Q. Li, Y. He, P. Petersson, Z. Li, M., Aldén, and X.-S. Bai, "Visualization of multiregime turbulent combustion in swirl-stabilized lean premixed flames," Combust. Flame 162, 2954 (2015).

${ }^{93}$ A. J. Aspden, M. S. Day, and J. B. Bell, "Lewis number effects in distributed flames," Proc. Combust. Inst. 33, 1473 (2011).

${ }^{94}$ M. Oberlack, H. Wenzel, and N. Peters, "On symmetries and averaging of the $G$-equation for 
Publishingpremixed combustion," Combust. Theory Modelling 5, 363 (2001).

${ }^{95}$ A. N. Lipatnikov and V. A. Sabelnikov, "Some basic issues of the averaged $G$-equation approach to premixed turbulent combustion modeling," Open Thermod. J. 2, 53 (2008).

${ }^{96}$ V. A. Sabelnikov and A. N. Lipatnikov, "Rigorous derivation of an unclosed mean G-equation for statistically 1D premixed turbulent flames," Int. J. Spray Combust. Dynamics 2, 301 (2010).

${ }^{97}$ M. L. Frankel and G. J. Sivashinsky, "The effect of viscosity on hydrodynamic stability of a plane flame front," Combust. Sci. and Technol. 29, 207 (1982).

${ }^{98} \mathrm{Z}$. Chen and Y. Ju, "Theoretical analysis of the evolution from ignition kernel to flame ball and planar flame," Combust. Theory Modelling 11427 (2007).

${ }^{99}$ Ya. B. Zel'dovich, G. I. Barenblatt, V. B. Librovich, and G. M. Makhviladze, The Mathematical Theory of Combustion and Explosions (Consultants Burea, New York, 1985).

${ }^{100}$ A. R. Kerstein and W. T. Ashurst, "Propagation rate of growing interfaces in stirred fluids," Phys. Rev. Lett. 68, 934 (1992).

${ }^{101}$ M. Chertkov and V. Yakhot, "Propagation of a Huygens front through turbulent medium," Phys. Rev. Lett. 80, 2837 (1998).

${ }^{102}$ B. I. Shraiman and E. Siggia, “Scalar turbulence,” Nature 405, 639 (2000).

${ }^{103}$ A. Tsinober, An Informal Conceptual Introduction to Turbulence (Springer, Heidelberg, 2009).

${ }^{104}$ V. R. Kuznetsov and V. A. Sabelnikov, "Combustion characteristics of mixed gases in a strongly turbulent flow," Combust. Explos. Shock Waves 13, 425 (1977).

${ }^{105}$ K. R. Sreenivasan, R. Ramshankar, and C. Meneveau, "Mixing, entrainment and fractal dimensions of surfaces in turbulent flows," Proc. R. Soc. London A 421, 79 (1989).

${ }^{106}$ O. Chatakonda, E. R. Hawkes, A. J. Aspden, A. R. Kerstein, H. Kolla, and J. H. Chen, “On the fractal characteristics of low Damköhler number flames," Combust. Flame 160, 2422 (2013).

${ }^{107}$ G. I. Taylor, "Statistical theory of turbulence. IV. Diffusion in a turbulent air stream," Proc. R. Soc. London A 151, 421 (1935).

${ }^{108}$ J. O. Hinze, Turbulence (McGraw Hill, New York, 1975) 2nd ed.

${ }^{109}$ P. Clavin, "Dynamical behavior of premixed flame fronts in laminar and turbulent flows," Prog. Energy Combust. Sci. 11, 1 (1985). 


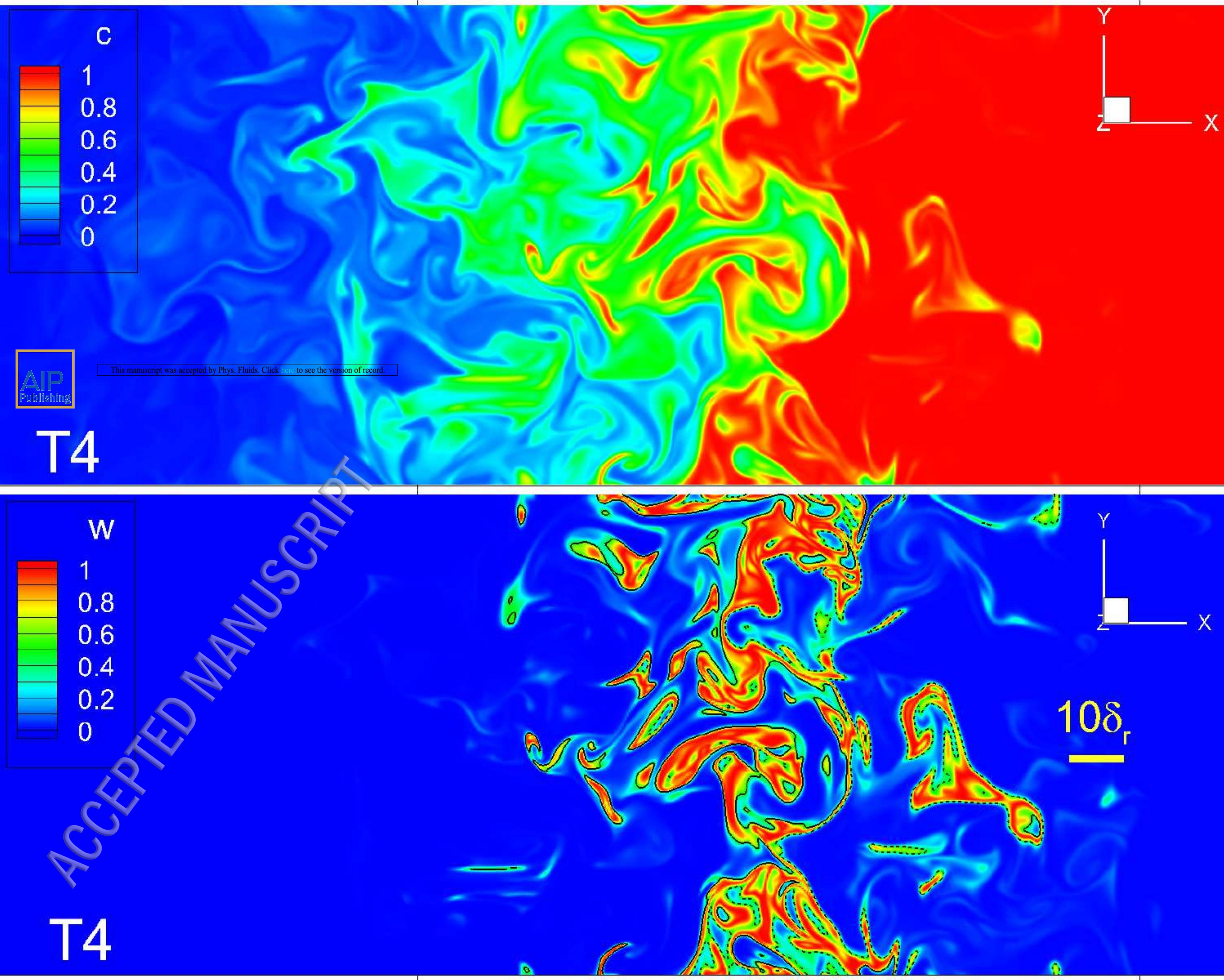




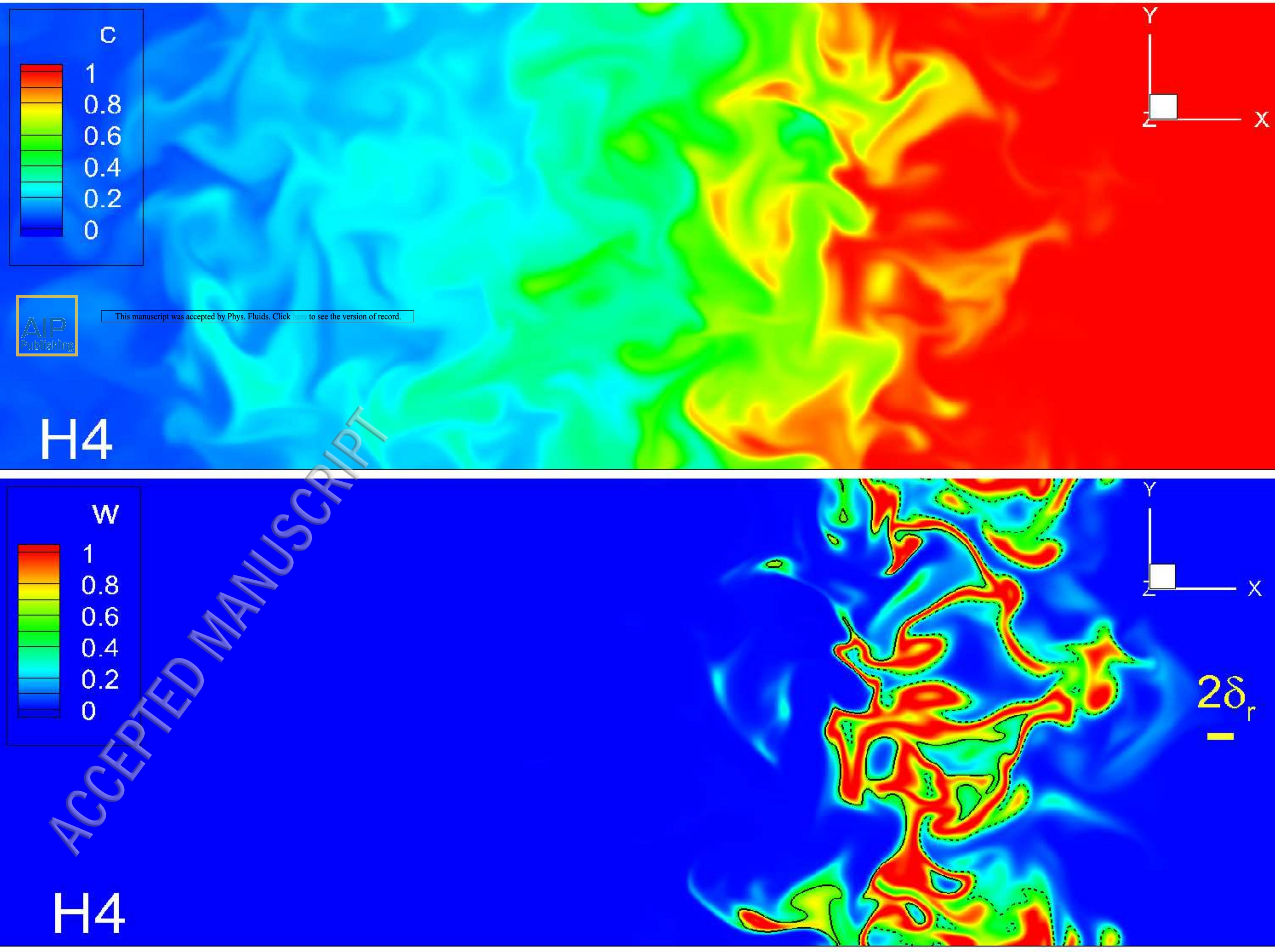




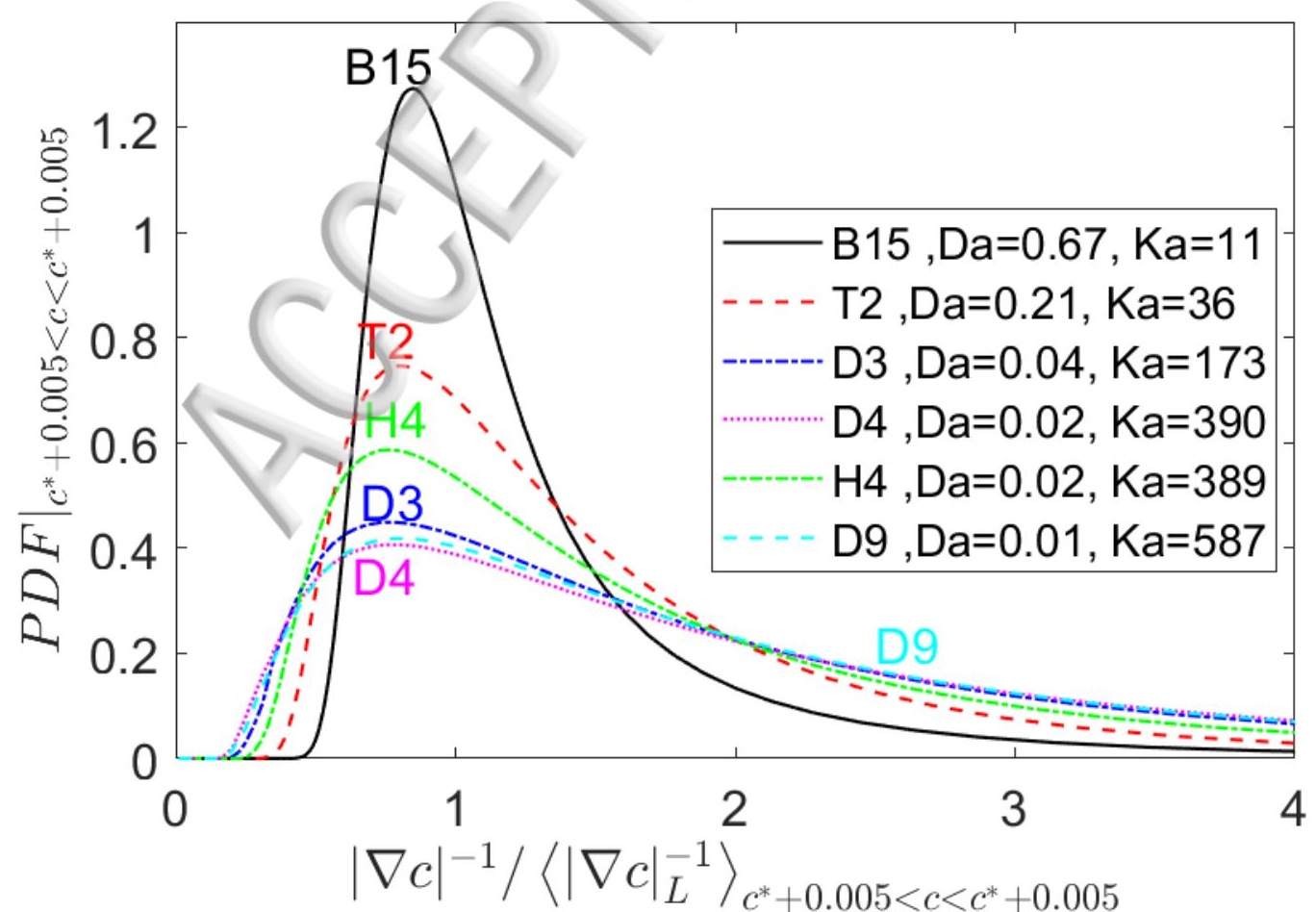




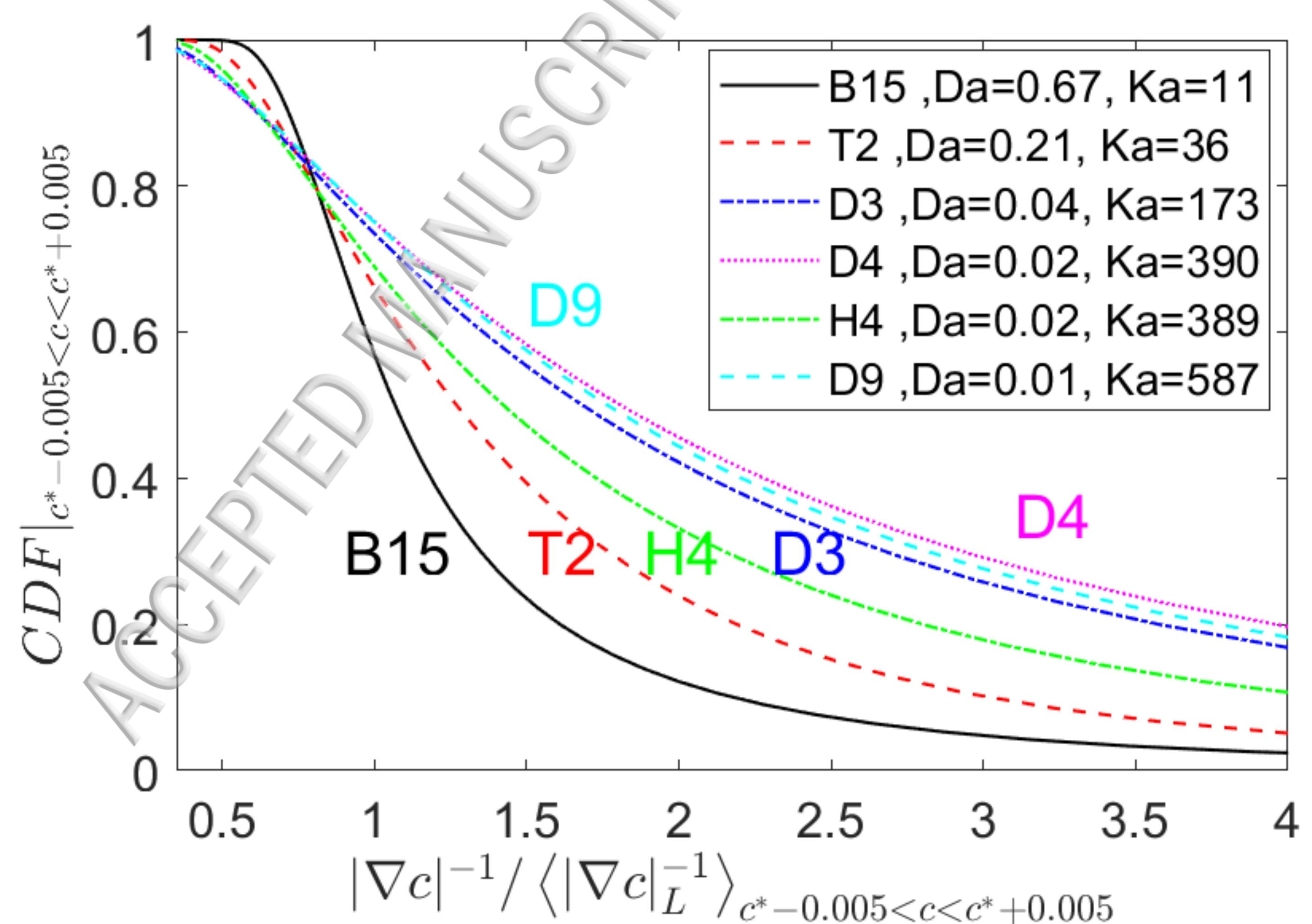




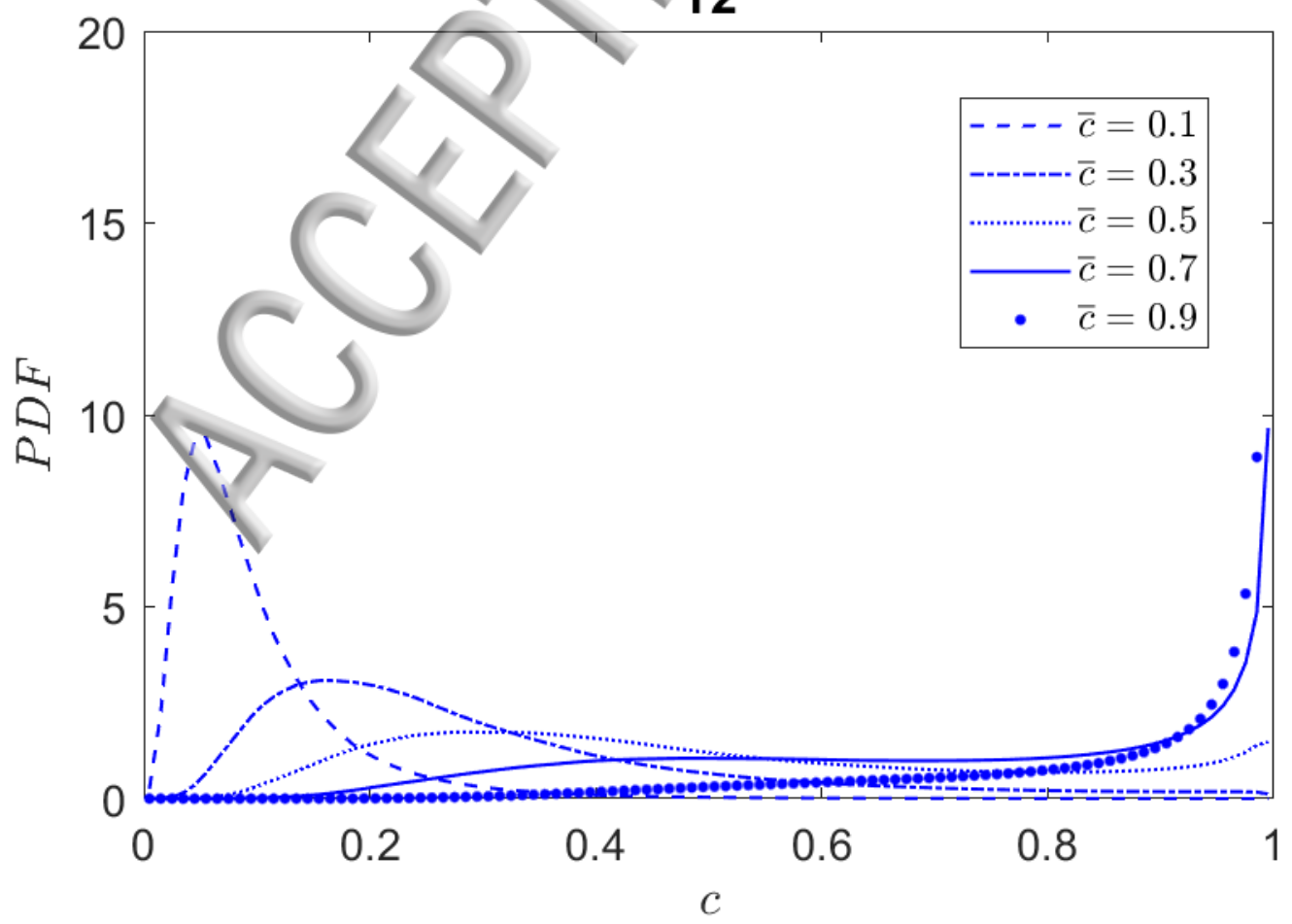


D9

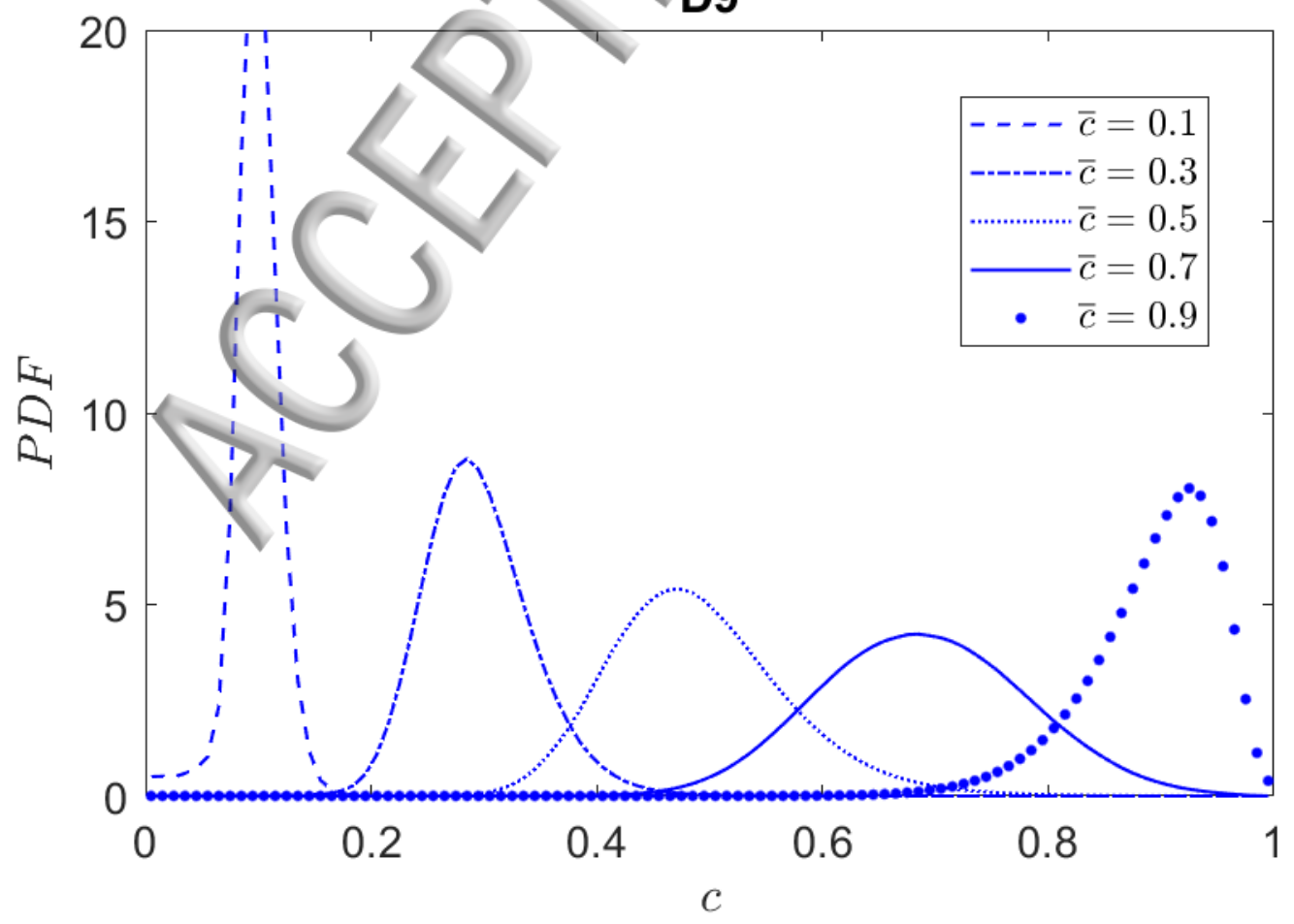




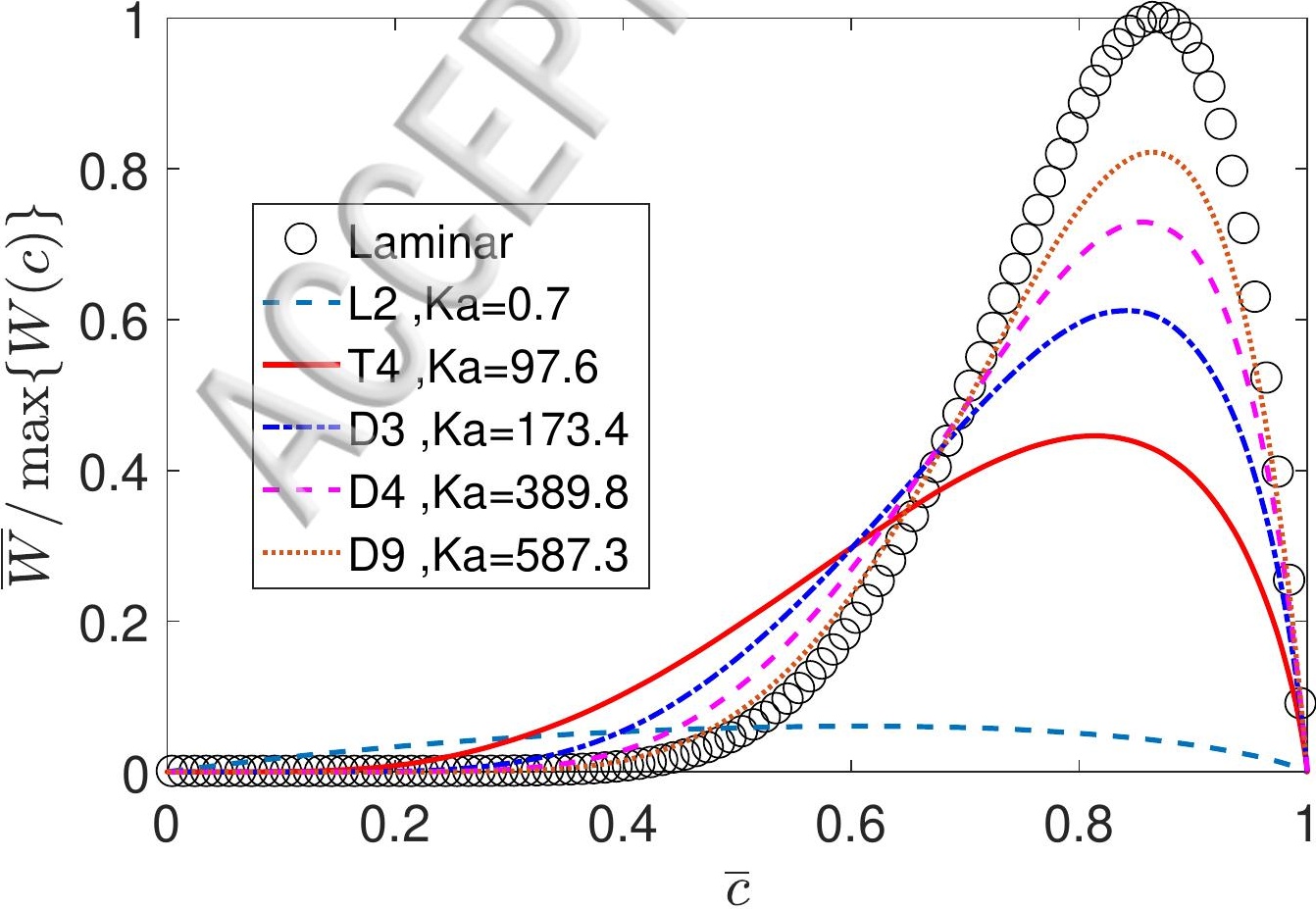


